\title{
Traditional Chinese Medicine and Clinical Pharmacology
}

\author{
Anita Chen Marshall
}

\section{Contents}

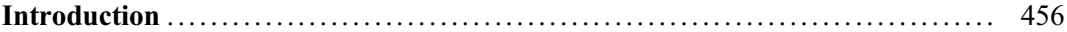

Principles and Practices of Traditional Chinese Medicine (TCM) ............ 457

Applications of Five Elements Theory ............................ 458

TCM Diagnostic Methods ........................................... 458

TCM Treatments ................................................. 461

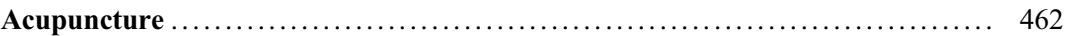

Chinese Herbal Medicine ............................................ 463

Characteristics in Herbal Medicine .................................... 467

Contemporary Applications of TCM Herbs in Clinical Pharmacology ......... 467

Herbal Medicine Formulation ..................................... 469

Interactions Between Herbs .................................... 469

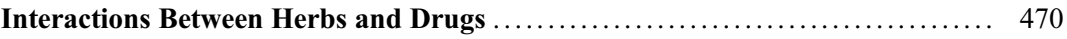

Herbal Processing, Quality Assurance, and Standardization .............. 471

Herbal Medicine and Pharmaceuticals: Toxicity, Adverse Reactions, and Mortality

A. C. Marshall $(\bowtie)$

Sequoia Healing Center for Acupuncture and Herbal Medicine, Alameda, CA, USA

American College of Traditional Chinese Medicine

(ACTCM), California Institute of Integral Studies (CIIS),

San Francisco, CA, USA

Bastyr University, Seattle, WA, USA

John A. Burns School of Medicine, University of Hawaii,

Honolulu, HI, USA

e-mail: dranitamarshall@gmail.com 
Antimicrobial Resistance and Herbal Medicine ....................... 474

TCM and Infectious Disease ................................... 474

Advancements in Cancer Research with TCM ......................... 476

Network Pharmacology and Systems Biology: The Next Generation of Herbal

Medicine ................................................. 477

TCM and Clinical Pharmacology In Silico ........................... 479

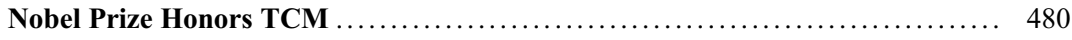

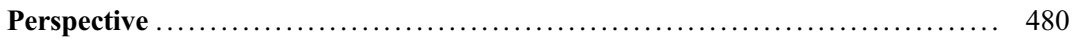

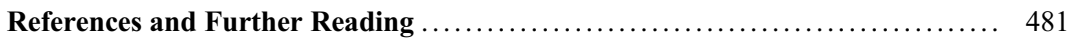

\section{Abstract}

Traditional Chinese Medicine (TCM) represents thousands of years in treatment protocols for health, healing, and longevity and has evolved into a complex healthcare system. The following chapter is designed to give a basic concise overview of TCM principles and practices, in relation to clinical pharmacology, and focuses on mutually relevant areas of interest. Much as society learned to harness electrical currents to empower machinery, TCM theories of energetic patterns help to empower the functionality of the human body. The major theories include the vital life force, known as Qi, Yin and Yang, Five Elements, herbal medicine, multivariate diagnostics, syndrome differentiation, and acupuncture, among others. In TCM, there is a direct relationship to the energy of nature and the energetic patterns within the human body and the human consciousness. This comprehensive approach is gaining momentum in clinical and network pharmacology, as new technologies emerge regarding TCM collaborative compounds versus singular chemical constituents in treatment modalities. Of major importance to clinical pharmacology are recent TCM evidenced-based empirical studies, correlations, and interactions between herbs and pharmaceuticals, updated data on toxicity, adverse reactions, quality assurance, and herbal medicine standardization. Global concerns over antimicrobial resistance to drugs have put a spotlight on TCM herbal medicines as alternatives with greatly reduced resistance factors. From ancient archives to the
Nobel Prize to a formal endorsement by the World Health Organization as a global healthcare system, TCM has widened the lens of modern science. It offers the wisdom and experience of millennia to inspire the think tank of today's medicine and pharmacology and tomorrow's generations of integrative healthcare advancements.

\section{Introduction}

Traditional Chinese Medicine (TCM) is renowned and respected among the world's time-honored systems of wellness and healing. Although legend and mythology allude to TCM origins as far back as 5000 years BCE, it was during China's Shang dynasty (1766-1122 BCE) TCM was systematized among the highly esteemed traditional herbalists and healing practitioners of the imperial courts. The first authenticated text to reference the concept of Yin and Yang (阴阳), which is the most important and distinctive theory of TCM, was the “Book of Changes" (Yi Jing 易經), dating back to around 700 BCE. Together with the concept of Qi (氣), vital life force, also translated as Chi (pronounced as "chee"), they provide the basic foundation for TCM's comprehensive mind, body, and spirit ideology and practice.

On May 25, 2019, TCM was officially adopted into the 11th version of the International Statistical Classification of Diseases and Related Health Problems (ICD). This document represents the formal global classification of diseases by the 
World Health Assembly, the governing body of the World Health Organization (WHO). This official compendium has recognized TCM as a traditional healthcare system and endorsed it to its 129 global member states. Excerpted from the WHO website regarding the ICD-11: "ICD is the foundation for the identification of health trends and statistics globally, and the international standard for reporting diseases and health conditions. ICD-11 was submitted to the Seventy-second World Health Assembly in May, 2019 and, following endorsement, Member States will start reporting using ICD-11on1 January 2022."

This chapter has been carefully written to inform and inspire readers to evaluate better the benefits of TCM in relation to conventional medicine and clinical pharmacology. TCM has a rich lineage of traditional knowledge bases and skill sets and has survived millennia of medical and scientific evolution to be acknowledged as a major contributor to twenty-first-century global healthcare. The compelling wisdom that TCM offers modern medicine is the primary position of prevention above treatment and discovering each individual's unique blueprint of wellness.

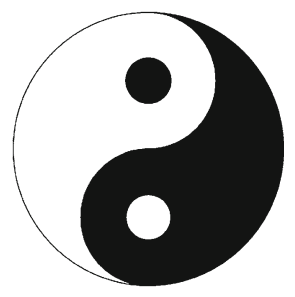

The law of yin and yang is the natural order of the universe, the foundation of all things, the mother of all changes, the root of life and death...

The Yellow Emperor's Inner Classic (Huang Di Nei Jing-黄帝內經)

\section{Principles and Practices of Traditional Chinese Medicine (TCM)}

One of the most important theoretical concepts of TCM is Yin (阴) and Yang (阳) that were recorded in the "Book of Change" (Yi Jing 易經), dating from $700 \mathrm{BCE}$. The iconic Yin/Yang symbol represents opposite properties and functions but has complementary qualities (see Table 1). Yin and Yang are mutually dependent and supportive of each other; one cannot survive without the other. Extreme Yin will transform itself into Yang, and extreme Yang will transform itself into Yin. There is Yin (small dark circle) within Yang (large white area above) and Yang (small white circle) within Yin (large dark area below), as illustrated in the Yin/Yang symbol.

All treatment goals are centralized around balancing the Yin and Yang through harmonizing the body, mind, and spirit. Internally, this is accomplished through a combination of the healing properties of food, herbs, acupuncture, moxibustion, Tui Na massage, cupping, meditation, and Taichi and Qigong exercises. In addition, there is correcting of harmful external environmental factors: expelling toxins, avoiding extreme temperatures, and eliminating physiological and emotional stressors. In TCM, a state of wellness and well-being can only be achieved when there is a state of equilibrium. What may be viewed in metaphysics as the harmonic convergence is expressed in conventional medicine as homeostasis and in TCM as ultimate balance.

TCM has been a champion of energetic medicine for millennia, because its underlying principles and practices stem from a foundational philosophy of the pervasive energetic force that permeates all of nature. In TCM, Qi is the key which opens the meridian channels or energy pathways and provides movement throughout the interconnected domains of health and healing. In the Yellow Emperor's Inner Classic (Huang Di Nei Jing, 黄帝内经), the most revered ancient text of TCM, there is a description of the principles of Yin and Yang, the blood vessels, and meridians, also known as channels (Jing Mai 经脈). Qi flows within the body, as well as the five Zang organs (五脏), the small intestine, gall bladder, large

Table 1 Courtesy of Anita Chen Marshall, DAOM, Pharm D, LAc

\begin{tabular}{l|l|l|l|l|l|l|l|l|l}
\hline \multicolumn{7}{l}{ Correspondences of Yin and Yang } & & & \\
\hline Yang & Sun & Sky & Brightness & Male & Activity & Fire & Rapid & Posterior & Strength \\
\hline Yin & Moon & Earth & Shade & Female & Rest & Water & Slow & Anterior & Weakness \\
\hline
\end{tabular}


intestine, stomach, and urinary bladder; six $\mathrm{Fu}$ organs (六腑), the heart, liver, lungs, spleen, kidneys, and pericardium; and associated acupuncture points (Xue Wei 穴位).

In addition to the concept of Yin/Yang in TCM, this text also introduces the Five Elements Theory (五行). This theory encompasses all natural phenomena, as they symbolically relate to human nature. As expressed in the Yellow Emperor's Inner Classic, the Five Element activities of Wood (liver, gallbladder), Fire (heart, small intestine), Earth (spleen, stomach), Metal (lung, large intestine), and Water (kidney, urinary bladder) are arranged in relationship sequences in which each element interacts with one another by generating, controlling, overacting, and insulting.

- Generating Sequence (normal): Each element generates another and is generated by another. Wood generates Fire, Fire generates Earth, Earth generates Metal, Metal generates Water, and Water generates Wood. Often, TCM elaborates on this reality of one element generating another, as evidenced in a motherchild relationship. For example, Wood is the child of Water, while Wood is the mother of Fire.

- Controlling Sequence (normal): Each element controls another and is controlled by another. For example, Wood controls Earth, Earth controls Water, Water controls Fire, Fire controls Metal, and Metal controls Wood. Another controlling example may be found in the following: Wood controls Earth, but Earth generates Metal, which in turn controls Wood. Therefore, this is a self-regulating sequence that keeps the body in balance. Excess controlling is perceived as "overacting" and can weaken other elements.

- Insulting Sequence (abnormal): It is the reverse order of the Controlling Sequence. Wood insults Metal, Metal insults Fire, Fire insults Water, Water insults Earth, and Earth insults Wood. This causes the body to go out of balance. When the element becomes excessive, it will insult and weaken the corresponding element and, over time, it will result in imbalances and pathological symptoms (Fig. 1).

\section{Applications of Five Elements Theory}

The applications of the Five Elements Theory in TCM are numerous, and the systems of correspondences are linked to many explicit phenomena and qualities in nature and the human body. These correspondences are inclusive of organs, tissues, tastes, directions, climates, colors, seasons, etc. TCM also recognizes the healing power of musical tones, which correspond to each of the five elements. These tones are Jue (角Wood), Zhi (徵Fire), Gong (宫Earth), Shang (商Metal), and $\mathrm{Yu}$ (羽Water). Just as music is composed of harmonic and resonant layers, these unique tones are intrinsic to the interrelated vibratory balance of the human body, mind, and spirit, which TCM understands are needed to be in harmony with each other to achieve a sense of balance and health. Considering the importance of these tonal qualities, TCM recognizes music therapy as a vital healing modality. Each food and herb in TCM has a certain taste which is related to one of the elements. The five tastes are sour for Wood, bitter for Fire, sweet for Earth, pungent for Metal, and salty for Water. Each of the tastes has a unique effect on the body. For example, the sour taste generates fluids and Yin; it has an astringent quality and, therefore, can control perspiration and diarrhea. As another example, bitter taste clears heat, sedates, and hardens. Table 2 outlines these integrated corresponding aspects.

\section{TCM Diagnostic Methods}

During the Song dynasty (960-1279 CE), TCM was fully systematized to include comprehensive diagnosis, disease symptom profile analysis, and multivariate treatment protocols. Together with the theory of Yin and Yang, the Five Elements Theory forms the essential philosophy and methodology of TCM. As mentioned previously, TCM practitioners use a combination of the theories of Yin/Yang and the Five Elements to identify the cause of each disease, excess, deficiency, or debility and develop a customized treatment protocol. In the course of this 


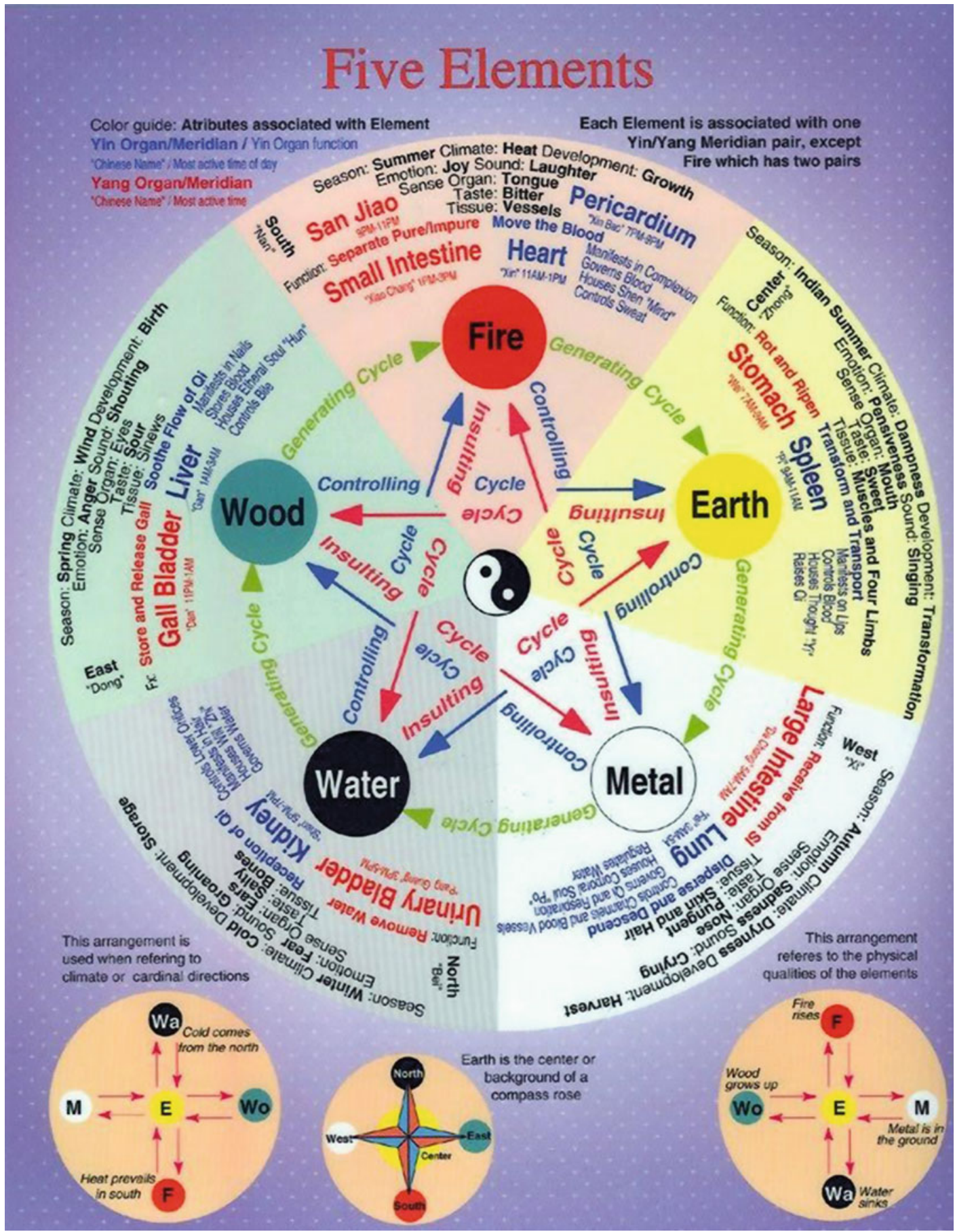

Fig. 1 Five elements chart. (Courtesy of Zdenek Zumr, MTCM/Introduction to Oriental Medicine through Charts and Graphs, 1994)

cohesive cycle of diagnosis from root causes to customized remedies, appropriate treatments are prescribed.
TCM uses the body's microsystems to treat the whole person effectively, mirroring a holographic perspective of each part reflecting the whole. This 
Table 2 Corresponding aspects of five elements theory. (Courtesy of Anita Chen Marshall, DAOM, Pharm.D., LAc)

\begin{tabular}{l|l|l|l|l|l}
\hline & Wood (木) & Fire $(火)$ & Earth $( \pm)$ & Metal (金) & Water (水) \\
\hline Yin organs & Liver & Heart & Spleen & Lung & Kidney \\
\hline Yang organs & Gallbladder & Small intestine & Stomach & Large intestine & Urinary bladder \\
\hline Sense organs & Eyes & Tongue & Mouth & Nose & Ears \\
\hline Tissues & Sinews & Vessels & Muscles & Skin & Bones \\
\hline Tastes & Sour & Bitter & Sweet & Pungent & Salty \\
\hline Colors & Green & Red & Yellow & White & Black \\
\hline Emotions & Anger & Joy & Pensiveness & Sadness & Fear \\
\hline Seasons & Spring & Summer & None & Autumn & Winter \\
\hline Directions & East & South & Center & West & North \\
\hline Colors & Green & Red & Yellow & White & Black \\
\hline Sounds & Shouting & Laughing & Singing & Crying & Groaning \\
\hline Odors & Rancid & Scorched & Fragrant & Rotten & Putrid \\
\hline Spirit & Ethereal soul & Mind & Thought & Corporal soul & Will power \\
\hline Climates & Wind & Heat & Dampness & Dryness & Cold \\
\hline Development & Birth & Growth & Transformation & Harvest & Storage
\end{tabular}

can be seen in various parts of the body reflected in multiple areas within the face, eyes, ears, hands, and feet. On a therapeutic level, TCM addresses these through acupuncture point manipulation. As an example: auricular acupuncture, hand and foot reflexology, etc.

While western medicine utilizes a stethoscope for detecting auscultations, TCM takes note of various sounds and pitches of the voice, coughing, breathing, wheezing, hiccupping, groaning, etc. In general, a loud sound reflects a full/excess pattern, while a weak sound is indicative of an empty/deficient pattern. TCM also takes into account the various odors emanating from the body. These odors can be related to different organ imbalances within the five elements system of correspondences, for example, rancid for the liver, scorched for the heart, sweet for the spleen, rotten for the lungs, and putrid for the kidneys. Halitosis indicates heat in the stomach and so on.

Examination and consultation in TCM are similar to taking a western medical history and complete physical. This process is extensive and can help to find the causative factors of the illness by determining the timing, environment, situations with living conditions, emotional and social issues, environmental exposures, etc. The most commonly asked questions are regarding symptom profiles, such as fever, chills, sweating, painful areas, urination and bowel movement, food and taste, thirst and drink, sleep patterns, and condition of eyes, ears, throat, and other body parts. There are specific questions for women regarding menstruation, discharges, pregnancy, and childbirth, as well as, for men, regarding virility and impotence, loss of strength, hair loss, hearing loss, and other targeted male issues.

Pulses: Of particular importance to TCM, which distinguishes it from allopathy, is the attention to various pulses, unique to major organs and meridians. Chinese pulse diagnosis is an effective tool to extract detailed information on the state of the internal organs and the corresponding meridians by palpating the radial artery on both wrists, dividing them into three areas and accessing them at three different levels: superficial, middle, and deep.

As important as the rate of pulses are the qualities of pulses, which can be described as fast, slow, strong, weak, thin, wiry, tight, choppy, slippery, floating, deep, etc. These pulse indicators give the TCM practitioner a clear view of underlying conditions. For example, a strong wiry pulse may indicate liver Qi stagnation and excess of stress; a slippery pulse indicates dampness within the meridians, and a floating pulse indicates allergy condition or cold symptoms.

Tongue: Another specialized TCM diagnostic area is the microsystem of the tongue. The 
practitioner takes careful note of the different shapes, colors, patterns, and coatings of the tongue, each giving specific details regarding the patient's condition. As treatments progress, multiple changes in the appearance of the tongue take place, indicating the efficacy of the chosen protocol.

A central principle of TCM diagnostic protocol is known as syndrome differentiation. This concept summarizes the interconnections of sensory diagnostic methods of observation, auditory, olfactory, consultative, pulse-taking, and palpation, in conjunction with pathological signs of the body, including the unique stages of disease development. Combined, these diagnostic methods are useful in identifying disease etiology, location, nature, and the relationship between the disease factors and a functional, balanced Qi. After evaluating these properties through diagnostic methods, the combined information leads to a syndrome differentiation analysis that aids in determining the most effective therapeutic protocol to eliminate pathogenic profiles.

\section{TCM Treatments}

TCM is a holistic and complex treatment system containing several treatment modalities that are utilized to promote healing and wellness. These modalities are acupuncture, herbal medicine, moxibustion, acupressure, Tui Na massage, cupping, nutrition and lifestyle consultation, meditation, and Taichi and Qigong exercises to strengthen the body and promote Qi and blood circulation (Fig. 2).

Moxibustion involves the heating of acupuncture points with smoldering mugwort herb (known as moxa). Moxibustion stimulates circulation, counteracts cold and dampness in the body, and promotes the smooth flow of blood and Qi. This safe, noninvasive technique may be used alone, but it is generally used in conjunction with acupuncture treatment.

Acupressure uses the same acupuncture points with gentle but firm pressure of the fingers and hands on the surface of the skin to increase the Qi and blood circulation, stimulating the body's natural curative abilities. Acupressure is the most effective method for treatment of tension-related ailments by using the power and sensitivity of the human hand, relaxing the muscles and relieving tension.

Tui Na massage means "pushing grasping" and is a powerful form of Chinese medical bodywork. Based on the same Oriental medical principles as acupuncture, Tui Na seeks to improve the flow of Qi through the meridian channels and is particularly effective for conditions involving muscles, tendons, and joints, such as structural misalignment, orthopedic problems, and sports injuries. It can also be used to treat internal diseases.

Cupping is an ancient technique, in which a special cup is applied to the skin and held in place by vacuum-like suction. The suction draws superficial tissue into the cup, which may be left in place or moved along the body. Cupping brings fresh blood to the area and helps improve circulation. Traditional cupping, sometimes referred to as fire cupping, uses heat to create a suction inside of glass cups. In modern times, plastic cups are used with a small vacuum pump to create suction, and small rubber cups that can be hand manipulated, without heat, have been introduced for convenience and safety.

Dietary therapy and nutritional consultation refers to selection of foods to help enhance patient's overall health, based on their patterns of disharmony. According to TCM, everyone has a different body constitution, temperament, and health conditions, which change at different ages. Diet and nutrition can lead the body back to balance by avoiding foods that aggravate imbalances. This concept correlates well in today's modern medicine where a large percentage of cancers and other degenerative diseases are known to be caused by improper diet and nutritional deficiencies.

Implicit in any diet is consideration of the agronomic factors in growing and cultivating plants with toxic pesticides, herbicides, and fungicides, as well as commercial farming of fish, poultry, and cattle with GMO feed, added hormones, and antibiotics, adding to toxic overload of the environment. These were nonexistent 
Fig. 2 TCM treatment modalities. (Courtesy of Anita Chen Marshall, DAOM, Pharm D, LAc)

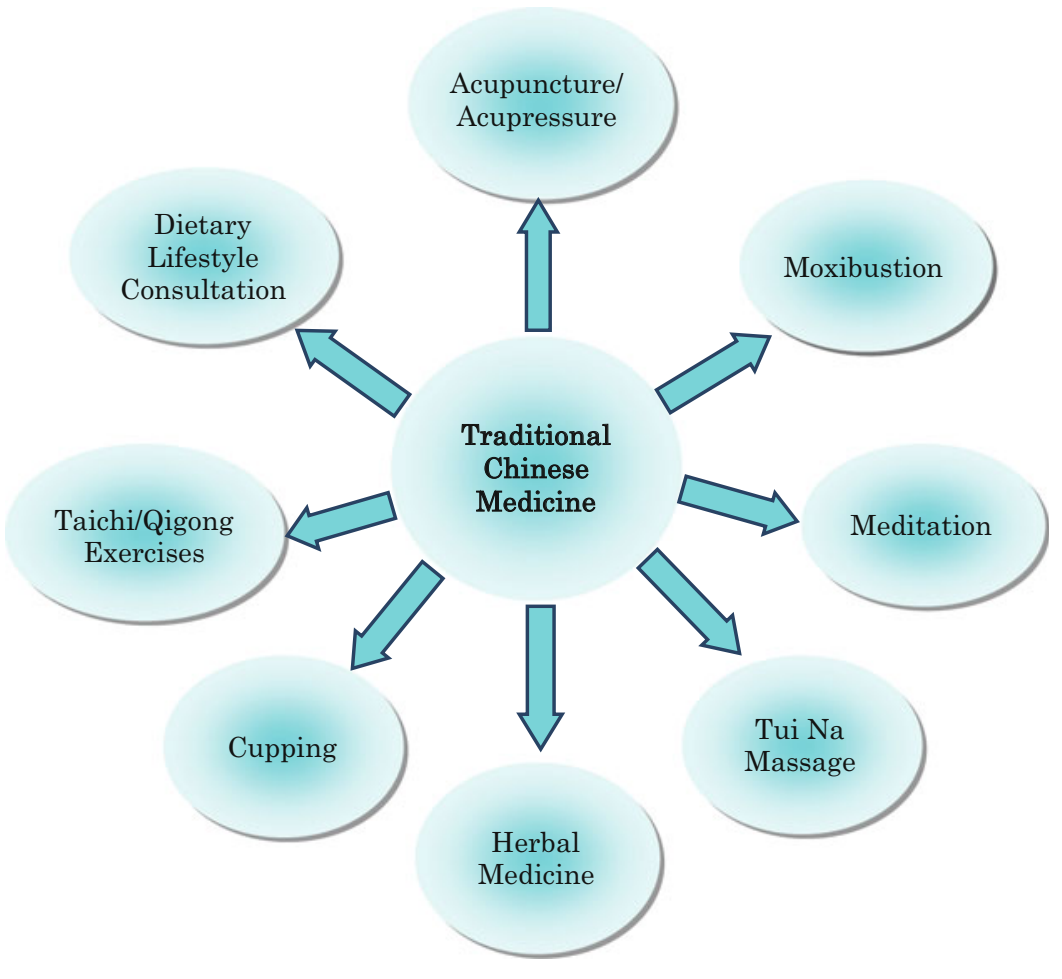

thousands of years ago when TCM was first developed but, in a modern polluted global environment, represent a very real challenge to contemporary human, animal, and environmental health.

A definitive landmark study, published in The Lancet in April 2019, involved a compilation of statistics (1990-2017) on morbidity and mortality related to dietary factors on a global scale. The findings indicate that 11 million deaths in 2017 were attributable to dietary risk factors showing suboptimal diet is responsible for more deaths than any other risks globally, including tobacco smoking. TCM acknowledged the vital importance of diet and nutrition thousands of years ago and continues to rank food therapy as the first line of treatment (Afshin et al. 2019).

Qigong means "life force energy cultivation" by practicing deep breathing techniques along with certain forms of movement, some of which mimic animals in nature. Medical Qigong is used as a treatment modality to promote, preserve, and restore health. The full benefits of Qigong exercises can be achieved when using correct posture and diaphragmatic breathing with conscious mental direction in a daily regimen. Qigong is often used to help reduce blood pressure, stress, and anxiety and promote restful sleep and wellbeing, in addition to eliminating common aging degeneration and promoting longevity.

Taichi, a form of Qigong, is a slow movement system of set forms of exercise based on Qigong principles of breathing, meditation, strength, and balance. Hua Tuo (华佗) is a reputed physician during 140-208 CE, who gained fame for his profound abilities in acupuncture, moxibustion, and medical Da Yian (大雁) exercises. It was his keen observation of the animal kingdom that lead him to develop the Wuqinxi (Exercise of the Five Animals, 五禽戲) from studying movements of the tiger, deer, bear, monkey, and crane.

\section{Acupuncture}

In TCM, there are 12 major meridians with more than 700 acupuncture points distributed bilaterally throughout the entire body and 2 central 
meridians. The anterior meridian is Ren Mai (Conception Vessel 任脈), and the posterior meridian is Du Mai (Governing Vessel 督脈).

Acupuncture is a treatment modality performed by inserting very fine sterile needles, not much thicker than a human hair, in specific locations on the skin, known as acupuncture points. The depth of needle insertion depends on the disease being treated and the location of the acupuncture point. For example, a shallow insertion is generally used for treating disease at the superficial level, i.e., initial stage of common colds and skin and muscle conditions. By contrast, deeper needle insertion is generally required for treating internal disease, e.g., organs and bones.

Acupuncture stimulation regulates the flow of Qi within the energy pathways, which is referred to as meridian or channel that interconnects throughout the entire body. The practice of acupuncture affects the endocrine and central nervous system, including responses from both the sympathetic nervous system (Yang) and the parasympathetic nervous system (Yin) to achieve equilibrium and promote the body's innate healing ability (Fig. 3).

According to ancient Chinese texts, each organ has a two-hour ultimate activity period per day. When the energy of a meridian is not flowing well, it will exert certain symptoms, and the symptoms become exacerbated. By paying attention to the timing of the symptoms, a TCM practitioner can detect the underlying root cause of the problem.

For example, patients with asthmatic conditions often awaken coughing with an asthma attack between 3 and 5 AM (Lung meridian period) during the pre-dawn time. The TCM doctor can utilize the time of the meridian to design the treatment protocol. For example, the best time to tonify the Kidney meridian deficiency is 5-7 PM. If the Liver meridian (1-3 AM) is excessive, one can use the Small Intestine meridian period (1-3 PM) to sedate the Liver meridian (Fig. 4) (Table 3).

According to the World Health Organization publication reporting the effectiveness of acupuncture as evidenced in reviews and analysis of clinical trials (WHO 2002) and the 2017 Acupuncture
Evidence Project from Australia, acupuncture has been recognized with significant clinical results for: allergic rhinitis, chemotherapy-induced nausea and vomiting (with anti-emetics), chronic low back pain, headache (tension and chronic), knee osteoarthritis, migraine prevention, postoperative nausea and vomiting and postoperative pain. These conditions show the strongest evidence for acupuncture effectiveness, while there are hundreds of more conditions that show a positive response (McDonald and Janz 2017). In addition, acupuncture has been acknowledged in the Journal of Integrative Medicine in 2017, involving thirteen professional institutes and clinical venues in the US, as having been proven to be a nonpharmacological option as a solution to the opioid crisis in America (Fan et al. 2017).

\section{Chinese Herbal Medicine}

Nature is the most prolific and elaborate laboratory on earth, and Chinese herbal formulations have held historical influence for nearly 5000 years. China has developed into the largest population in human history, over 1.4 billion as of 2019 , noted by the United Nations. This grand vision on the ultimate human experience gives TCM the perfect empirical model for human trials that has proven highly effective over several millennia and now is center stage in the global arena of pharmaceutical research and development.

Central to TCM is herbology and the cultivation, combination, and application of herbal remedies. TCM makes use of herbs and herbal decoctions to strengthen organ function and rebalance the metabolism. A deep base of knowledge and a keen sensitivity to the essence of various herbal components give the TCM practitioner a precise method of generating a healing effect that reaches beyond the chemical composition and physical properties of the herbs.

The practitioner chooses the herbal formula that best complements the individual's unique symptom profile and the body's energetic vibration, as determined through various diagnostic processes.

According to the World Health Organization (WHO), herbal medicines provide the basis of 


\section{Human body meridians}
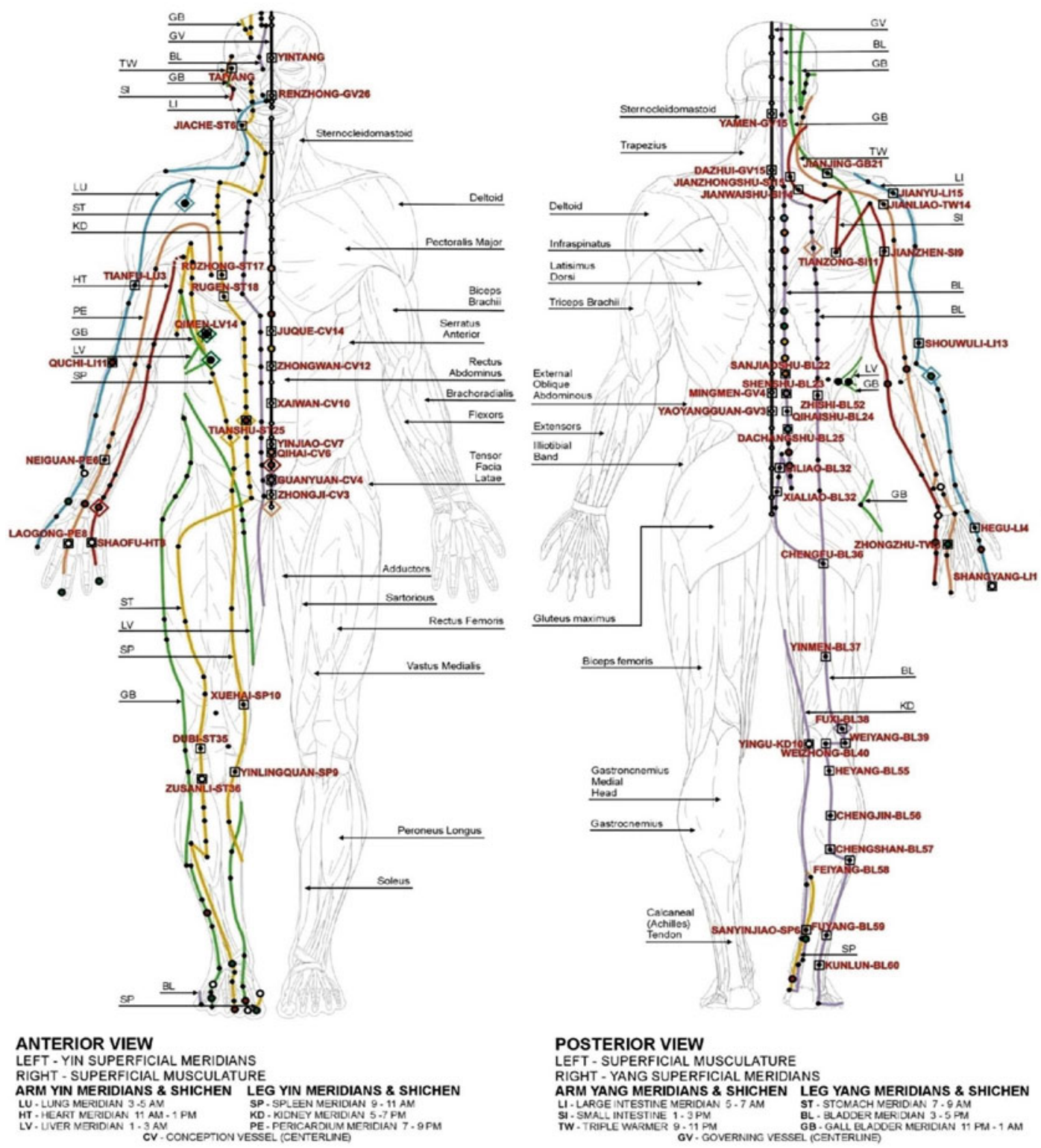

\section{POSTERIOR VIEW}

LEFT - SUPERFICIAL MUSCULATURE

RIGHT - YANG SUPERFICIALMERIDIANS

ARM YANG MERIDIANS \& SHICHEN LEG YANG MERIDIANS \& SHICHEN

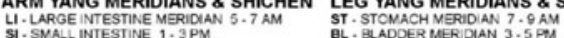

TW-TRIPLE WARMER 9.11 PM GB. GALL ELADOER MERIDIAN 11 PMA - 1 AM GV. GOVERAING VESSEL (CENTERLINE)
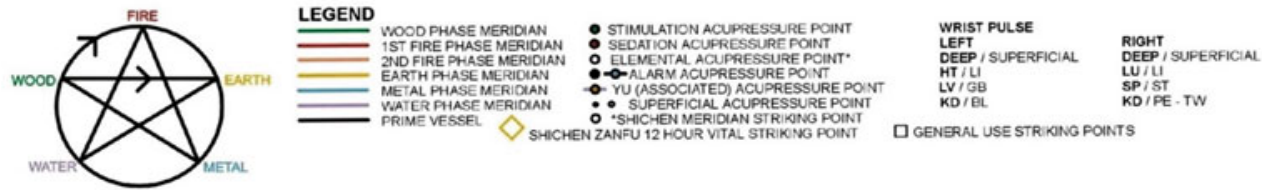

GENERAL USE STRIKING POINTS

Fig. 3 Meridian channels chart -Wikimedia. (Permission to use: Creative Commons License 4.0) 


\section{Yin Yang Clock}

$$
\begin{aligned}
& \text { Trace the gray spiral in clockwise direction and follow } \\
& \text { the timely progression of Qi within the body. } \\
& \text { Trace the flow of Qi on your body and } \\
& \text { see the continuity of the flow. }
\end{aligned}
$$

\begin{tabular}{|c|c|c|c|c|}
\hline Deepest Yin & & ive level of pene & Ion & Superficial Yang \\
\hline $\begin{array}{l}\text { Xue level } \\
\text { heat in the } \\
\text { blood level } \\
\text {-XS and } \\
\text { deficiency heat }\end{array}$ & $\begin{array}{c}\text { Ying level } \\
\text {-nutritive oi level } \\
\text {-deficiency heat } \\
\text { likely }\end{array}$ & $\begin{array}{l}\text { Four Heat Levels of } \\
\text { Wen Bing } \\
\text { "Infectious Disease" }\end{array}$ & $\begin{array}{l}\text { Qi level } \\
\text {-first internal level } \\
\text {-alternating chills } \\
\text { and fever }\end{array}$ & $\begin{array}{l}\text { Wel level } \\
\text {-defensive } Q \mathbf{i} \\
\text {-simultaneous } \\
\text { chills and fever }\end{array}$ \\
\hline
\end{tabular}
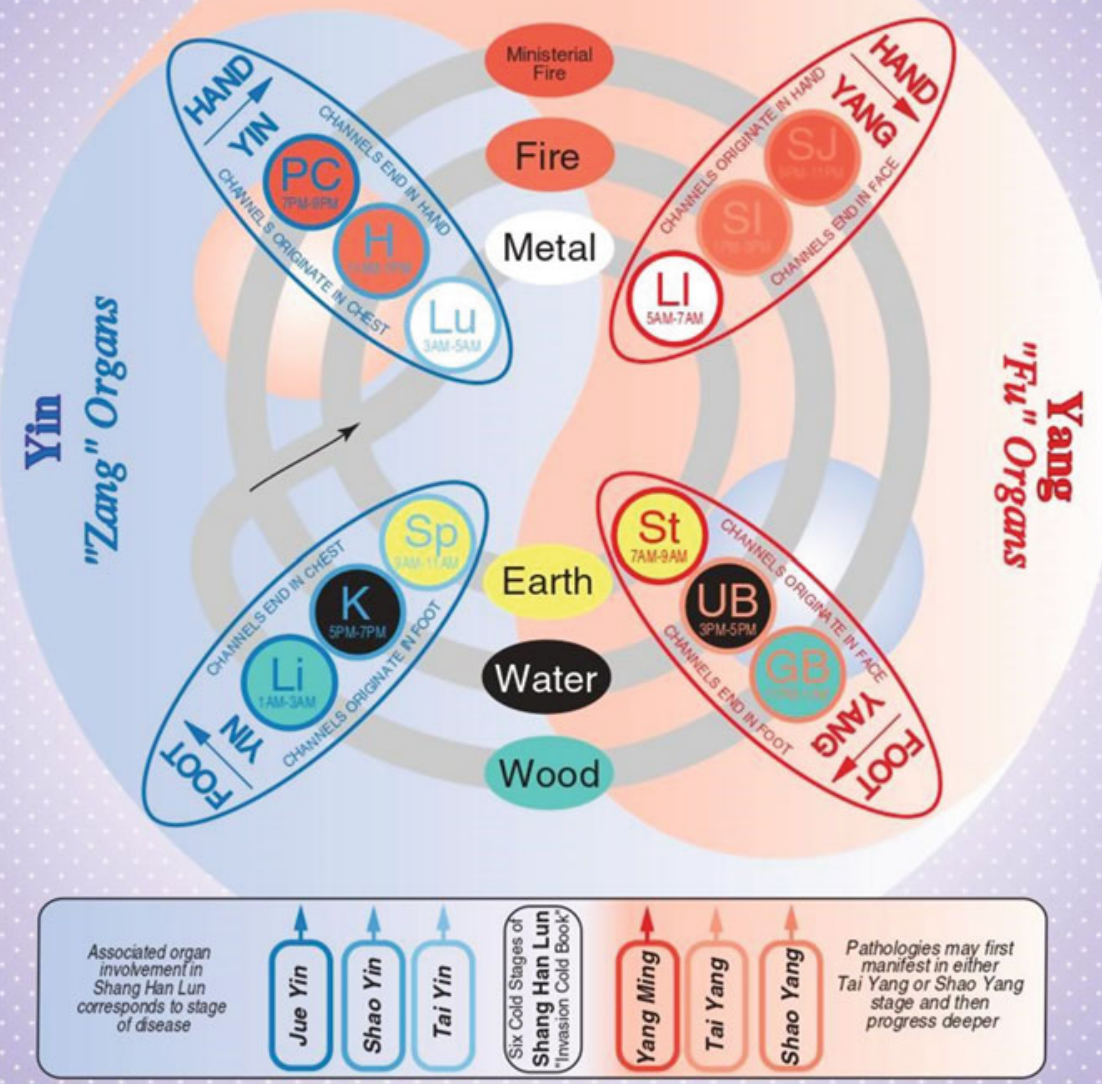

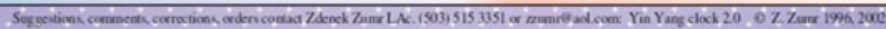

Fig. 4 Yin Yang clock. (Courtesy of Zdenek Zumr MTCM: Introduction to Oriental Medicine through Charts and Graphs, 1994) 
Table 3 Twelve meridian time flow chart. (Courtesy of Anita Chen Marshall, DAOM, Pharm D, LAc)

\begin{tabular}{l|l|l|l}
\hline Zang organs (Yin meridians) & Hours & Fu organs (Yang meridians) & Hours \\
\hline Lung (Hand Tai Yin) & 3-5 AM & Large Intestine (Hand Yang Ming) & $5-7$ AM \\
\hline Spleen (Foot Tai Yin) & 9-11 AM & Stomach (Foot Yang Ming) & $7-9$ AM \\
\hline Heart (Hand Shao Yin) & 11 AM-1 PM & Small intestine (Hand Tai Yang) & $1-3$ PM \\
\hline Kidney (Foot Shao Yin) & $5-7$ PM & Urinary bladder (Foot Tai Yang) & $3-5$ PM \\
\hline Pericardium (Hand Jue Yin) & 7-9 PM & San Jiao (Hand Shao Yang) & $9-11$ PM \\
\hline Liver (Foot Jue Yin) & 1-3 PM & Gallbladder (Foot Shao Yang) & 11 PM-1 AM \\
\hline
\end{tabular}

primary healthcare for more than four billion people on a global level, comprising over half of the world's population.

It is estimated that nearly $85 \%$ of traditional medicine modalities involve plant extracts that have been the foundation of modern pharmacology for centuries. There is a bounty of intrinsic and extrinsic properties that TCM recognizes as complementary to the innate healing capacity of the human metabolism. Herbs have biochemical compositions of active and buffering agents that synergistically balance each other. Some herbs are well known as food sources, while others are purely medicinal.

In contrast to the targeted medications of conventional allopathy that are largely developed and prescribed to ameliorate specific metabolic dysfunctions or specific symptoms, TCM herbal formulations work in collaboration with one another to achieve a systemic rebalancing. Each herb plays a unique role in the ultimate goal of achieving equilibrium, which is specific to each person. By combining the expertise of multiple herbal formulations, TCM can precisely address an individual patient's particular set of issues and develop a custom-designed healing protocol.

Volumes of herbal formulas, medical applications, and variables on TCM practices for individualized protocols can be found in numerous historical texts from various pivotal TCM authors and practitioners, and many still are circulated and researched today. Respect is due to the ancestral authors, whose wisdom and intuitive knowledge laid the groundwork for TCM, then and now. All TCM practitioners are grateful to pass on this heritage and offer their personal contributions for the next generation.

1. Huang Di Nei Jing (黃帝內経) The Yellow Emperor's Inner Classic (300 BCE) is the most revered of TCM's ancient texts. It is believed to have been the product of hundreds of separate writings gathered into two texts: Suwen (Basic Questions 素問) and Lingshu (Spiritual Pivot 靈樞). It is often regarded as the theoretical foundation of TCM and its diagnostic methods. It includes the dynamics between forces of nature (Yin/Yang) and the universal instinct to regain and maintain balance and harmony.

2. Shen Nong Ben Cao Jing (神农本草经) Divine Husbandman's Classic of the Materia Medica (200 CE). This manuscript detailed over 300 herbs according to thermal property, taste, toxicity, dosage, and forms. It was owing to this manuscript and this era that many widely used herbal remedies are still utilized today, such as Ma Huang (Herba Ephedrae) for asthma relief, Dang Gui (Radicis Angelica sinensis) for regulation of menstruation, and Huang Lian (Rhizoma coptidis) for cessation of diarrhea.

3. Shang Han Za Bing Lun (伤寒杂病论), Treatise on Cold Pathogenic and Miscellaneous Diseases (150-219 CE), was written during the Eastern Han Dynasty by physician Zhang Zhong Jing (张仲景). The main focus of the manuscript was on treatments for epidemic infectious diseases, causing fevers. In addition, he wrote Jin Gui Yao Lue (金贵要 略) Essential Prescriptions of the Golden Cof$f e r$, which focuses on treatment for internal diseases.

4. Yao Xing Ben Cao (药性本草) Materia Medica of Medicinal Properties (600 CE). This manuscript elucidated the complex subjects of combination, reaction, taste, temperature, toxicity, function, primary clinical application, processing, and preparation. This is also an important text in light of it being the 
first attempt to collaborate herbal empirical data, clinical application, and classical TCM theory as a cohesive compendium.

5. Shi Liao Ben Cao (食疗本草) Materia Medica of Diet Therapy (700 CE) was written by Meng Shan (孟詵;). It emphasized the importance of diet and the use of 227 herbs as therapeutic agents.

6. Ben Cao Gang Mu (本草纲目) The Grand Materia Medica (1578 CE) was compiled by Li Shi Zhen (李時珍). A formidable encyclopedia, it contains information on 1892 substances: 1173 from plants, 444 from animals, and 275 minerals. In addition, it proposed 11,000 herbal formulas for specific ailments.

7. Ben Cao Gang Mu Shi Yi (本草綱目拾遺) (1765 CE) was compiled by Zhao XueMin (趙 學敏). This text provided a complete description of over 700 natural substances catalogued for the first time.

8. Zhong Hua Ben Cao (中华本草) The Chinese Herbal (1999 CE) was compiled by the Chinese State Administration of TCM involving over 500 scholars/experts from at least 60 academic and research institutions, and took 10 years to complete. This monumental textbook consists of 10 volumes, totaling 9,282 pages, with 8,534 illustrations and 8,980 Chinese Materia Medica (CMM) monographs. There is a condensed 2-volume version available. The modern literature covered is up to 1994. It is the most extensive documentation of CMM to date.

\section{Characteristics in Herbal Medicine}

In TCM, there is an acknowledgment of variables that are known as characteristics. Precise differentiation of the pattern of symptoms is correlated to the distinguished properties of each herb in herbal medicine. These characteristics reference specific value sets that are uniquely descriptive and, therefore, aid the practitioner in determining the herbal choices which correlate best to a series of symptoms and individual patient constitution.

The characteristics of Chinese Herbal medicine are divided according to taste, thermal property, direction, and channel affiliations. While different characteristics have separate evaluative criteria, each of them must be taken into account while formulating the herbal medicine. In exploring these options, for instance, choosing the correct taste, but the wrong thermal property or an inappropriate meridian, could result in an ineffective treatment. If the patient has a heat condition, they should not be given herbs with hot or warm thermal property. By doing so, it would not only be ineffective but may also worsen the heat condition. The directional affinity is used to guide the herb's function toward the treatment area. For example, Huang Qin (Radix Scutellariae) clears heat in the upper jiao (chest) area and, therefore, can be used to treat lung or liver diseases. Du Huo (Radix Angelicae Pubescentis) moves in a downward direction, useful for both treating and guiding other herbs in treatment for musculoskeletal disorders of the lower body (Fig. 5).

\section{Contemporary Applications of TCM Herbs in Clinical Pharmacology}

Historically, herbal medicine was the single most effective medicinal option available to humans. Food and plant derivatives were the centerpiece of treatment protocols before the advent of industrialization, synthesis of chemicals, and pharmaceutical development. Today, there is resurgence of interest toward potent phytochemicals and other compounds from plants to discover more effective and less toxic therapeutic modalities for infectious and degenerative diseases, as well as cancer treatments.

TCM is currently at the forefront of global medical research and is evolving exhaustive databases of the complexity of chemical compounds found within classic TCM herbal decoctions that are being currently investigated in pharmaceutical studies. Over 71,000 entries, between 1945 and 2019 (abstracts not available until the 1980s), are noted for TCM in Pubmed.gov from the US National Library of Medicine and the National Institutes of Health.

Additionally, there are over 3000 studies, specific to certain herbal compounds that are used in infectious and degenerative diseases, many of 


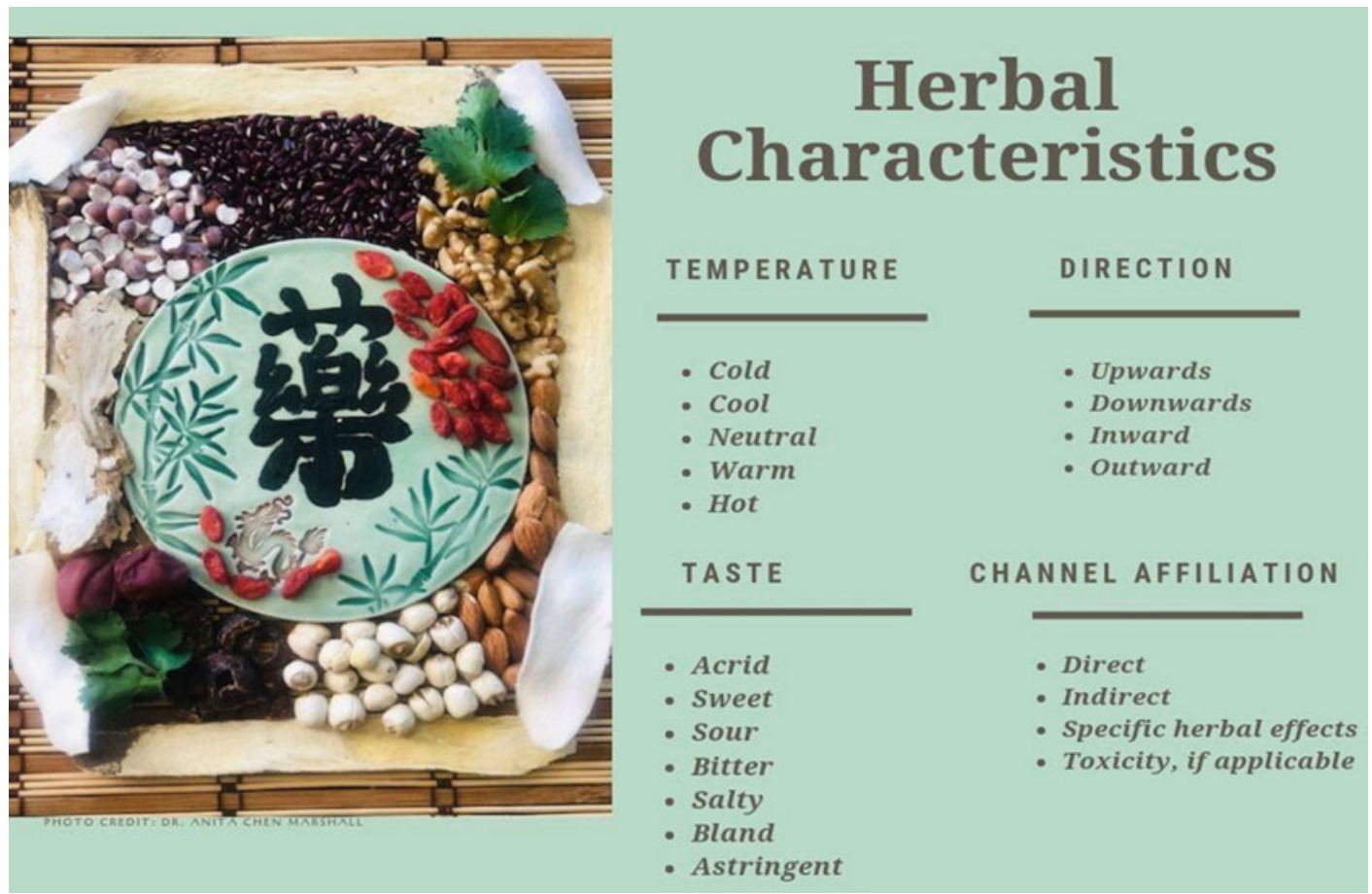

Fig. 5 Herbal characteristics. (Courtesy of Anita Chen Marshall, DAOM, Pharm D, LAc)

which have developed states of resistance, despite standard antibiotics and pharmaceuticals.

Just as herbal medicine is a cornerstone of TCM, pharmaceuticals have become the mainstay of conventional medicine. Major pharmaceutical companies in the USA, Europe, and Asia are premiere research and development sources for these drugs. Much of the history of pharmaceuticals has its origin with herbal traditions, such as TCM. An examination of this history and how herbs and drugs have become synonymous for medicine in different eras can be quite enlightening. Current statistics indicate an estimated wealth of 300,000-500,000 plant species on the planet, of which only $10 \%$ has been cultivated and developed for medicinal purposes. Biodiversity efforts are being proposed to help protect these plants from extinction and to sustain their natural global habitats (Rates 2001).

Herbal compounds have a well-documented history for treating diseases. Between 35,000 and 70,000 plant species have been screened for medicinal compounds, and over
200 modern drugs owe their origins to the plant kingdom (Veeresham 2012). It was reported by Fabricant and Farnsworth (2001) that $80 \%$ of 122 plant-derived drugs were related to their original ethnopharmacological purposes.

Many of the commonly known herbal compounds used today, such as curcumin from Jiang Huang (Rhizoma Curcumae Longae, turmeric), Sheng Jiang (ginger), and Guan Ye Lian Qiao (Hypericum perforatum, aka, St. John's wort), are utilized for their anti-inflammatory, antidepressant, and antiemetic properties. Curcumin has become a major component in cancer research in the decades between 1999 and 2019 with over 21,000 studies cited in the PMC - National Library of Medicine and National Institutes of Health databases. Many of these are landmark studies indicating curcumin's significance in cancer prevention, cancer stem cell elimination, and advanced cancer therapies.

It is postulated that $60 \%$ of antitumor and anti-infectious drugs, already on the market or 
under clinical trials, are of plant origin (Cragg 1997). Other plant compounds including cannabinoids, muscarine, physostigmine, yohimbine, forskolin, and colchicine have been clinically used in numerous pharmacological and biomedical studies (Williamson et al. 1996). Studies have shown a TCM herbal formula may be applied for several western classifications of pathologies.

For example, Liu Wei Di Huang Wan is a classic TCM formula used for the TCM pattern "Yin deficiency and tonify Kidney meridian." Recent studies have proven that this formula is effective for multiple diseases, including metabolic syndrome, diabetes, and cardiovascular disease, based on clinical experiences and pharmacological studies (Jiang 2005).

Clinical pharmacology now involves a multidisciplinary cohort of sciences including botany, chemistry, toxicology, anthropology, agronomy, and biotechnology, all of which have advanced pharmacology and improved the standards of drug development. Principles in TCM that adhere to a philosophy of interrelated networks in nature and the human physiology and psychology are now being seriously investigated in network pharmacology and systems biology.

A modern pharmacological approach, bioactivity-guided fractionation, is essential in the isolation of active substances. Because TCM often prescribes a multivariate decoction of several herbs, designed to assist a core biological response, research in standardization may not always rely on a singular compound or focus of action, but on a synergistic result, which has both pharmacodynamic and pharmacokinetic properties.

For instance, Radix Ginseng, well reputed in TCM circles, is a prime example in which the whole plant or its saponin fractions are more active than the isolated compounds (Hamburger and Hostettman 1989). As TCM and network pharmacology continue to join efforts toward cataloguing herbal compounds and designing an international platform of standardization, herbal medicines will be more acceptable to empirical review parameters of clinical study.

\section{Herbal Medicine Formulation}

Chinese herbal medicine recognizes an optimal therapeutic effect is achieved through identifying and analyzing the intricate nuances of the specific disorder in relationship to the person's unique character and constitution. Single herbs may be prescribed in the event of a clear diagnosis without additional complexities. However, as most pathological conditions are aggregations of imbalances, a system of complementary herbal remedies, custom-designed for the individual and the pattern of symptoms, has evolved from ancient days.

In Huang Di Nei Jing (The Yellow Emperor's Inner Classic), the basic principle of formulating an herbal medicine is described as the system of Jun Chen Zuo Shi (Chief, Deputy, Assistant, Envoy). Each herb performs an important and essential function within the formula.

This general concept has been expanded from the original, but the basic principle is still being followed in modern times.

Jun Yao (Chief Herb) is the most important component in each formula. It is used to treat the key disease or symptom, which is used at a large dose to maximize the effect. Chen Yao (Deputy Herb) reinforces the effects of the chief herb and also treats the associated disease or symptoms. Zuo Yao (Assistant Herb) reinforces the chief or deputy herb to treat the disease or directly treat the secondary symptoms. It is used to counteract the toxicity or to minimize the undesirable strong effects of the chief and deputy herb. Shi Yao (Envoy Herb) acts as a channel-guiding herb to direct the formula to the affected area of the body and also to harmonize all of the herbs within the formula, which is usually used in small doses.

\section{Interactions Between Herbs}

Although TCM herbal medicine has thousands of years of history attesting to its efficacy and safety, there are defined situations of toxicity that TCM practitioners are trained to understand and address. These guidelines contain six herb/herb interaction categories: 
1. Xiang Xu (mutual accentuation), Xiang Shi (mutual enhancement).

Example: combination of Shi Gao (gypsum) and Zhi Mu (Rhizoma Anemarrhenae) to "clear heat and purge" fire.

2. Xiang Wei (mutual counteraction) Xiang Sha (mutual suppression), Xiang Wu (mutual antagonism).

Example: combination of Lai Fu Zi (Semen Raphani) and Ren Shen (Radix Ginseng), in which the effect of the latter herb is decreased. Lai Fu Zi is used as antidote for Ren Shen overdose (symptoms: palpitation, bloating, tremor, distress).

3. Xiang Fan (mutual incompatibility). Example: eighteen incompatibles (Shi Ba Fan) Gan Cao (Radix Glycyrrhizae) is incompatible with Gan Sui (Radix Euphorbiae Kansui), Da Ji (Radix Euphorbiae seu Kaoxiae), Yuan Hua (Flos Genkwa), and Hai Zao (Herba Sargassum). Wu Tou (Radix Aconiti) is incompatible with Chuan Bei $\mathrm{Mu}$ (Bulbus Fritillariae Cirrhosae), Zhe Bai Mu (Bulbus Fritillariae Thunbergii), Gua Lou (Fructus Trichosanthis), Ban Xia (Rhizoma Pinelliae), Bai Lian (Radix Ampelopsis), and $\mathrm{Ba} \mathrm{Ji}$ (Rhizoma Bletillae). Li Lu (Radix et Rhizoma Veratri) is incompatible with Ren Shen (Radix Ginseng), Bei Sha Shen (Radix Glehniae), Nan Sha Shen (Radix Adenophorae), Ku Shen (Radix Sophorae Flavescentis), Dan Shen (Radix Salviae Miltiorrhizae), Xuan Shen (Radix Scrophulariae), Bai Shao (Radix Paeoniae Alba), Chi Shao (Radix Paeoniae Rubra), and Xi Xin (Herba Asari).

In conjunction with these categories of interactions, there are two levels of cautions and contraindications that are important: safety and effectiveness factors. These include interaction of the specific herbal natures with the specific symptoms of the condition and interaction of herbs directly with other herbs. While most herbs have been proven safe individually or in combination, certain herbal combinations have created adverse side effects and reactions.

\section{Interactions Between Herbs and Drugs}

As people are increasingly taking medications and herbal supplements, especially in the geriatric population, the potential of herb and drug interactions is becoming a growing concern for healthcare professionals, as well as the general public. There are two types of interactions: pharmacokinetic and pharmacodynamic. Pharmacokinetic interactions refer to the fluctuations in bioavailability of herb and drug molecules in the body, as a result of changes in absorption, distribution, metabolism, and elimination. Pharmacodynamic interactions refer to the fluctuations in bioavailability of ingested substances, as a result of synergistic or antagonistic interaction between herb and drug molecules.

Herbs also are known to exhibit numerous interactions with drugs. Duplicating similar reactions of herbs and drugs could cause overdose or ineffectiveness of drugs in the patient's metabolism. Following are descriptions of herbal functions which may interfere with or exacerbate biochemical reactions of specific classifications of drugs.

Guan Ye Lian Qiao (Hypericum perforatum), aka St. John's wort, is commonly used as an OTC supplement for depression and it is a liver enzyme inducer, which will lower the blood level of other medications if given together, leading to decreased therapeutic effect. It may also cause serotonin syndrome, if given concurrently with Selective Serotonin Reuptake Inhibitor (SSRI) drugs. Another herb, Chai Hu (Radix Bupleuri), should be used with caution with the drug interferon, as this herb has shown increased risk of acute pneumonitis. The herb may over stimulate neutrophils to release granulocytes elastase and oxygen radicals which may cause damage to lung tissues.

Physicians and clinical pharmacologists may be interested in exploring possibilities of TCM herbal medicines that offer similar bioactive properties as pharmaceutical drugs, yet with fewer to no adverse reactions.

Understanding both the chemical compatibility and incompatibility between herb and drug functions can develop options in research and treatment protocols that may provide safer and more effective modalities. Especially in major cardiovascular 
areas, where multiple drugs are often prescribed to achieve one effect, such as modulating hypertension or anticoagulation, TCM herbs may be more targeted to achieve the same results with a single herbal compound. Also, in areas of diabetes, depression, and anxiety, TCM herbal medicines have proven to be effective alternatives to more toxic multiple drug regimens. As with all active substances that can alter metabolic functions, caution should be used when prescribing any herb or drug that could be contraindicated. Table 4 displays certain interactions of herbs and drugs that should be considered in any combined treatment protocol.

\section{Herbal Processing, Quality Assurance, and Standardization}

The herbal extracts can be derived from the raw herbs through a solvent, such as grain alcohol, or an alcohol-free solvent, such as vegetable glycerin. However, the herbal decoction is the most

Table 4 Herb functions and drug interactions. (Courtesy of Anita Chen Marshall, DOAM, PharmD, LAc)

\begin{tabular}{l}
\hline Herb pharmacological functions \\
Elevate blood pressure and stimulate heart muscle \\
Sympathomimetic: Ma Huang (Herba Ephedrae), Qing Pi \\
(Pericarpium Citri Reticulatae Viride), Zhi Shi (Fructus \\
Aurantii Immaturus), Zhi Ke (Fructus Aurantii) \\
Hypertensive: Gan Cao (Radix Glycyrrhizae) \\
Positive inotropic: ChenPi (Pericarpium Citri Reticulatae), \\
Fu Zi (Radix Aconiti Lateralis Praeparata), Ren Shen \\
(Radix Ginseng), Wu Zhu Yu (Fructus Evodiae) \\
Note: \\
Hypotensive: Gou Teng (Ramulus Uncariae cum Uncis), \\
Di Long (Pheretima), and Ginkgo biloba (Folium Ginkgo) \\
may cause hypotension when used with antihypertensive \\
drugs \\
Improve blood circulation, inhibit platelet aggregation \\
Dan Shen (Radix Salviae Miltiorrhizae), Ji Xue Teng \\
(Caulis Spaatolobi), San Qi (Panax Notoginseng), Tao Ren \\
(Semen Persicae), Pu Huang (Pollen Tyhpae) \\
((He, LS et al. 2016)
\end{tabular}

Drain damp effect (increase urine output)

Fu Ling (Poria), .Zhu Ling (Polyporus), Che Qian Zi

(Semen Plantaginis), Ze Xie (Rhizoma Alismatis), Bai Zhu

(Rhizoma Atractylodis Macrocephalae)

Lower blood sugar
Zhi Mu (Radix Anemarrhenae), Shi Gao (Gypsum
Fibrosum), Shan Yao (Rhizoma Dioscorea), Huang Qi
(Radix Astragalus), Xuan Shen (Scrophularia ningpoensis),
Cang Zhu (Rhizoma Atractylodis)

Calm the mind (shen) sedative

Suan Zao Ren (Semen Ziziphi Spinosae), Yuan Zhi (Radix Polygalae), Chai Hu (Radix Bupleuri), Tien Ma (Rhizoma Gastrodiae), Ye Jiao Teng (Caulis Polygoni Multiflori), He Huang Pi (Cortex Albiziae), Fu Shou (Fructus Citri Sarcodactylis)
Drug interactions

\section{Antihypertensive drugs:}

Caution: may increase risk of hypertension

Calcium channel blockers: amlodipine (Norvasc), nifedipine (Procardia, Adalat), efonidipine (Landel), felodipine (Plendil)

Beta-blockers: metoprolol (Lopressor), labetalol (Normodyne, Trandate), propranolol (Inderal), carvedilol (Coreg)

ACE inhibitors: lisinopril (Prinivil, Zestril), benazepril, (Lotensin), captopril (Capoten)

Angiotensin receptor blockers: losartan (Cozaar), valsartan (Diovan)

\section{Anticoagulant and antiplatelet drugs}

Caution: may lead to prolonged PTT and excessive bleeding. Examples: warfarin (Coumadin), heparin, apixaban (Eliquis)

\section{Diuretic drugs}

Caution: may lead to excessive fluid and electrolyte loss Examples: hydrochlorothiazide (Microzide), furosemide (Lasix), spironolactone(Aldactone)

\section{Antidiabetic drugs}

Caution: may cause hypoglycemia metformin (Glucophage), tolbutamide (Orinase), glipizide (Glucotrol), glyburide (Glynase, Micronase), pioglitazone (Actos), insulin

\section{CNS suppressant drugs}

Caution: may cause excessive drowsiness and sedation Opioids: morphine, codeine, hydrocodone, oxycodone Antidepressants: selective serotonin reuptake inhibitors (SSRI), fluoxetine (Prozac), fluvoxamine (Luvox CR), paroxetine (Paxil)

Antipsychotics: aripiprazole (Abilify), quetiapine (Seroquel)

Antiseizure: phenytoin(Dilantin), pregabalin (Lyrica) gabapentin(Neurontin)

Benzodiazepines: diazepam (Valium), Lorazepam (Ativan), Alprazolam (Xanax)

OTC sleep aids: valerian, kava, melatonin, diphenhydramine (Benadryl), doxylamine (Unisome) 
common dosage form for herbs in China. The herbs are presoaked in enough water to cover them for a period of time, depending on the nature of the herbs and purpose. The herbs are then cooked until the water boils and then let simmer for a period of time, between 30 and $60 \mathrm{~min}$, depending upon the particular blend of herbs, before the decoction (tea) is ready for consumption. The remainder of the herbs can be boiled the second time or discarded. In addition, a variation on the herbal decoction can be freeze-dried and condensed into granules. Herbal extracts and granules, similar to contemporary medications, are more convenient for patients to take than the customary traditional raw herbs.

The process of preparing the raw herbs can be complex in timing. Leaves and flowers take less time to decoct than barks and roots; therefore, they should be added to the decoction process last. $\mathrm{He}$ Shou Wu (Radix Polygoni Multiflori) should be decocted with black beans at least for 30 min to decrease the side effects of loose stools, diarrhea, and liver toxicity. Long-term consumption of $\mathrm{He}$ Shou $W u$ has been associated with liver toxicity. If discontinued, adverse reactions are reversible. However, if it is overdosed through unmonitored consistent consumption, cirrhosis or death may occur (Lei et al. 2015).

Many factors must be considered in the cultivation, concentration, toxicity/adverse reactions, and standardization of herbal medicines. These include geographical origin, varying processing and manufacturing techniques, as well as contraindications and compatibility with other herbs and pharmaceuticals (Zhang et al. 2017).

As language can be a challenging issue in identifying a Chinese herb, in the interests of international standardization, use of both the Chinese term and the binomial, including the Latin botanical name and/or pharmaceutical name and any specific genus, would help to establish a global consensus regarding the exact herb in question. Effective monitoring of herbal medicine safety will result from the collaboration of botanists, phytochemists, pharmacologists, and other major contributors to the cultivation, processing, and distribution of herbal medicines (Ekor 2014).
The accurate processing of herbal decoctions can reduce the toxicity of a few specific herbs. For example, improper preparation or overdose of $\mathrm{Fu}$ $\mathrm{Zi}$ (Radix Aconiti Lateralis Praeparata) is the main cause of possible adverse reactions. A person with a weak constitution may also have toxic reactions to $F u Z i$, even when taken in low doses. Patients who have a past history of cardiovascular disease, or are taking anti-arrhythmic medications, should take $F u Z i$ with extreme caution due to its strong cardiovascular effects. $F u Z i$ should be decocted for more than an hour until the roots are very soft to decrease strong undesirable side effects and toxicity. Gross overdose can be fatal.

Proper processing, concentration, and dosages are as relevant in clinical pharmacology. Quality assurance and manufacturing integrity are related to effectiveness and toxicity, as well as less regulated sources, such as the generic drug industry. Quality assurance is now a major issue regarding the safety standards and potency of drug manufacturing for both herbal medicines and pharmaceuticals.

Prime examples of this are the industry recalls in 2018-2019 of losartan and valsartan, the widely prescribed angiotensin II receptor blockers for treating hypertension. In addition, the popular OTC medication for heartburn, Ranitidine (Zantac), was recalled in October 2019. Generic manufacturers are under the FDA microscope since it was discovered that these drugs were contaminated with ingredients containing trace amounts of $N$-nitroso- $N$-methyl-4-amino butyric acid (NMBA) and other carcinogens. The genotoxic impurities may be caused by changes made in the active pharmaceutical ingredient (API) manufacturing process but could also came from the reuse of materials, such as solvents. The growing concern over adulterated products and contamination of the API in China and India has grown into a regulatory challenge as to how to improve quality while maintaining costeffectiveness.

Up to $70 \%$ of drug manufacturing plants in China and India are being rated as substandard: falsifying records, tainting products, and allowing unsanitary conditions (Bottle of Lies: The Inside Story of the Generic Drug Boom, book by 
Katherine Eban 2019). It appears that the pharmaceutical industry in the USA and Europe, which obtains $80 \%$ of its raw materials from subcontracted global sources, is finding itself in a challenging situation. Inspection of the raw materials and these offshore manufacturing facilities and processes is integral to the future of safer and more effective pharmaceuticals, as well as herbal medicines and nutraceuticals (Identifying the Root Causes of Drug Shortages and Finding Enduring Solutions (Duke-Margolis et al. 2018) Duke University and FDA presentation-Washington, DC, November 27, 2018).

\section{Herbal Medicine and Pharmaceuticals: Toxicity, Adverse Reactions, and Mortality}

TCM herbal medicine is often scrutinized and criticized by the scientific community for nonregulated issues of safety and efficacy, as well as lack of evidence-based, peer-reviewed studies. The interesting aspect of empiricism is that it is based on observation, induction, deduction, experimentation, and evaluation. TCM has a documented history of such empirical values in the development of its multivariate diagnostics, combinative herbal therapies, and intrinsic relationship with the philosophical and physiological factors of human nature within the entirety of nature. The isolation of critical active ingredients may often be misleading in verifying how nature navigates both in the environment and within living inhabitants.

As regards toxicity and adverse reactions to TCM herbal formulations, there are several encyclopedias and collected documents, some of which are being translated into English. These include the Pharmacopoeia of the People's Republic of China (PPRC), Toxic Chinese Materia Medica: Past and Present (Du Ju Zhong Yao Gu Jin Yong), Encyclopedia of Toxic Chinese Materia Medica (You Du Zhong Cao Yao Da Ci Dian), Toxic Drugs Herbal (Du Yao Ben Cao), and the Modern Toxicology of Chinese Materia Medica (Xian Dai Zhong Yao Du Li Xue).

China's State Administration of Traditional Chinese Medicine requires all TCM practitioners to obtain the education and Chinese medicine degree to be officially registered and practice legally. In the Traditional Medicine Strategy 2014-2023 report, the WHO addressed concerns regarding toxicity and adverse reactions to TCM herbal medicines to strengthen safety protocols of TCM herbal products by global practitioners. The International Organization for Standardization (ISO) is in the process of developing quality standardization options for Chinese herbal medications, inclusive of raw materials, processing, and finished product quality assurance. These efforts will be beneficial in the regulation and standardization of the globalized marketing of TCM herbal medications (Zhou et al. 2019).

The concepts of toxicity and adverse reactions are no strangers to TCM, historically. In the Qing dynasty (1644-1911 CE), Herbal Lihai authored by Ling Huan identified a variety of species of medicinal plants in the order of three aspects: harm, benefit, and therapy. The harmful side effects and contraindications were listed in the beginning, indicating that TCM practitioners recognized possible adverse effects of herbal remedies, long before the age of clinical pharmacology (Zeng and Jiang 2010).

According to a 2014 report by Donald Light, $\mathrm{PhD}$, from the Edmond J. Safra Center for Ethics at Harvard University, the following information depicts the adverse reactions and deaths from prescribed drugs in the USA and European Union as gleaned from systemic hospital reviews: serious adverse reactions result in over 1.9 million hospitalizations a year, with 840,000 hospitalized patients being given drugs that cause serious adverse reactions for a total of 2.74 million serious adverse drug reactions annually. In addition, prescribed drugs result in an estimated 128,000 deaths annually in the USA alone, accounting the use of prescription drugs being the 4thleading cause of death, along with stroke. The European Commission estimates that adverse reactions from prescription drugs cause 200,000 annual deaths (Light 2014).

The International Regulatory Cooperation for Herbal Medicines (IRCH) and the WHO Collaborating Centre for International Drug Monitoring are focusing on the regulation, education, and 
standardization of traditional herbal medicine throughout the 129 member states. The 2019 adoption of TCM into the WHO ICD-11 calls for member states to begin reporting statistics and evidence-based data by January 1, 2022.

Once data is collected and analyzed, adverse reactions and morbidity and mortality rates for traditional herbal medicines may be discerned by equitable comparison of pharmaceuticals and TCM herbal medicines.

\section{Antimicrobial Resistance and Herbal Medicine}

Antimicrobial resistance is one of the most urgent health risks of our time and threatens to undo a century of medical progress, said Dr. Tedros Adhanom Ghebreyesus, WHO Director-General. Drug-resistant bacteria and viruses have adapted over several decades due to rampant use of antibiotics. There has been a monumental rise of refractory infections causing thousands of deaths annually worldwide.

The standard anti-pathogenic strategies of clinical pharmacology through modern medicine have reached a pivotal turning point in effectiveness, and a different approach needs to be investigated to reduce the current epidemic levels of antimicrobial resistance to drug therapies. There are a number of herbs that exhibit antibacterial and antiviral properties that may provide ways to decrease the development of antimicrobial resistance.

The WHO has enacted a new program to address resolution of this antimicrobial resistance dilemma entitled AWaRe (Access, Watch and Reserve). Released in October 2019, the program outlined a tool was developed by the WHO Essential Medicines List to contain the rising resistance and make antibiotic use safer and more effective. It classifies antibiotics into three groups: Access, Watch and Reserve to help emphasize their optimal uses and potential for antimicrobial resistance. (1) Access group: Have activity against common infections while have lower potential to develop resistance. (2) Watch group: Have higher resistance potential, should be prioritized as key target to be monitored. (3) Reserve group: Should be used sparingly and reserved for treatment of infections caused by multi-drug resistant organisms. It should be used only as a last resort.

The AWaRe campaign promoted by the WHO has mandated several actions areas including increasing the consumption of antibiotics in the Access group to at least $60 \%$ and reducing the use of the antibiotics in most at risk of resistance from the Watch and Reserve groups. Using Access antibiotics lowers the risk of resistance because they are "narrow-spectrum" antibiotics (that target a specific microorganism rather than several). They can be made available in generic forms and represent a less costly treatment option.

\section{TCM and Infectious Disease}

Conventional western medicine addresses infectious disease by a linear approach in suppressing and eliminating suspected viruses and bacteria that are presumed to be the primary causative factors. TCM and herbal medicine tend to offer a more comprehensive approach to infectious disease by applying the syndrome differentiation principle, a core feature of the TCM approach to individualized treatment. It has a vantage point in going beyond the viral and bacterial etiology concept to address the environment of the patient constitution in which the pathogens exist and the numerous influences affecting the patient's natural immunity. TCM protocols not only consider inhibition of pathogens but also, simultaneously, establish balance in the body and maintain organ recovery during severe and drug-resistant infection.

TCM acknowledges two syndromes, deficiency and excess, which occur commonly in severe infectious disease situations. In these cases, distinct treatments are used to dispel the pathogens by choosing either a deficiency or excess protocol which may include detoxification, clearing heat, cooling blood, removing blood stagnation, tonifying Qi, and nourishing Yin. 
By carefully appropriating the treatments to the syndrome differentiation, these methods can reduce inflammation, ameliorate circulation disorders, and aid in tissue fibrinolysis.

According to a comprehensive report on SARS (Severe Acute Respiratory Syndrome) from the WHO (2004), TCM has been shown to be an valuable adjunct therapy with western medicine for treatment of the epidemic outbreak of SARS in 2003. It should be applied at the beginning stage of the infection while considering a patient's individual differences and underlying conditions. The outbreak of Coronavirus Disease (COVID-19), originated from Wuhan, China in December 2019, spread rapidly in China and around the world, was declared pandemic by the WHO on March 11, 2020. There were 1,853,155 confirmed cases with 114,247 death reported by April 12, 2020. In February 2020, there were a few reports from China indicating that TCM and integrated therapies had shown advantages over western medicine alone in treating hospitalized patient with severe acute respiratory syndrome.

A report from Chen, JK. (2020), compiled and translated 3 different reports from China on How COVID-19 (2019-nCoV) is Currently Treated in China with TCM. It listed 4 different stages of treatment for COVID-19 in China. (1) Prevention phase (2) Influenza phase (3) Pneumonia phase (4) Recovery phase. Each phase presented a different clinical condition; therefore, it required different herbal formulas to treat the underlining conditions. The advantages of TCM treatments are primarily due to its effectiveness in relieving symptoms, decreasing lung inflammation, improving the degree of blood oxygen saturation, modulating immunological responses, reducing the required dosage of glucocorticoid and other Western medicines, decreasing the morbidity and mortality rate, and lowering the cost of treatment by shortening the duration of infectious stage. Since there is currently no effective treatment and no vaccine for COVID-19, the national authorities, health care workers and researchers should be inspired to explore the potential of a TCM integrative treatment approach.
The following categories of TCM herbs show antiviral, antibacterial, antifungal, and antiparasitic properties by their primary biochemical compounds and exhibit bioactivities in relation to the unique disease etiology:

1. Antiviral herbs: Jin Yin Hua ((Flos Lonicerae), Lian Qiao (Fructus Forsythiae), Ban lan Gen (RadixIsatidis), Da Qing Ye (Folium Isatidis), Huang Qin (Radix Scutellariae), $\mathrm{Yu}$ Xing Cao (Herba Houttuyriae), Xian Chun Ye (Folium Toonae Sinensis). Bioactivity: inhibition of viral replication and secretion of inflammatory cytokines from macrophages. May contain: tannins, flavonoids, polysaccharides, and alkaloids. Xian Chun Ye (Folium Toonae Sinensis) contains TSL-1 that selectively bind to SARS-CoV virus to inhibit the cellular entry of the virus into the host cells, it may be an important adjunct therapy in treating SARS-CoV (Chen CJ, et al. 2008), which is closely related to SARS-CoV2. More research should be done to investigate the effectiveness of adding this herb to the treatment for COVID-19.

2. Antibacterial herbs: Jin Yin Hua (Flos Lonicerae), Pu Gong Ying (Herba Taraxaci, dandelion), Da Huang (Radix et Rhizoma Rhei, rhubarb), Huang Lian (Rhizoma Coptidis, Coptis root), Di Fu $\mathrm{Zi}$ (Fructus Kochiae, broom cypress fruit, Kochia). Bioactivity: disruption of bacterial metabolism, structure, and functions; reversal of bacterial resistance; may contain organic acids, alkaloids, flavonoids, quinones, and volatile oils

3. Antifungal herbs: Ku Shen Gen (Radix Sophorae Flavescentis, sophora), She Chuang $\mathrm{Zi}$ (Fructus Cnidii, cnidium fruit), Di Fu $\mathrm{Zi}$ (Fructus Kochiae). Bioactivity: inhibition of fungal growth; may contain terpenoids and aldehydes, flavonoids, alkaloids, phenols, and volatile oils

4. Antiparasitic herbs: Bing Lang (Semen Arecae, betel nut), Qing Hao (Herba Artemisiae Annuae, wormwood). Bioactivity: induction of paralysis, blockage of nutrient absorption, inhibition of metabolism, and 
elimination of parasites; may contain alkaloids and phenols (Ma et al. 2019)

\section{Advancements in Cancer Research with TCM}

Cancer research has gained unparalleled momentum as the world population topped 7.7 billion in 2019 with over 14 million new cases of cancer being diagnosed annually and over 9.6 million cancer-related deaths reported worldwide. New cancer cases are expected to rise, inexorably, to 23.6 million by 2030. Cancer now has become the second leading cause of death globally (WHO 2018). These staggering statistics point to the imperative for all nations to focus on more effective protocols for cancer prevention and treatment.

Meta-analyses of randomized controlled studies involving TCM as adjunctive supportive cancer therapy show improved survival rates and performance status of chemotherapy and radiation treatments. TCM in partnership with clinical pharmacology offers promise for such research and development, both as adjuvant and primary cancer treatments. The following information has been excerpted from the chapter "Principles of Supportive and Palliative Care/Chinese Herbal Medicine as Adjunct Therapy in Patients with Lung Cancer" published in the textbook Modern Thoracic Oncology, Vol. 1, 2018.

Extensive evaluations have been conducted evaluating the specific mechanisms by which adjunctive Chinese herbs address the needs of cancer patients. Table 5 shows the bioactivities of herbs exhibiting therapeutic and palliative action (McCulloch et al. 2018).

One remarkable adjuvant herbal medicine development getting examined since 1999 is known as PHY906. The original herbal formula in TCM is referred to as Huang Qin Tang and is comprised of four herbs: Huang Qin (Radix Scutellaria), Bai Shao (Radix Paeoniae Alba), Gan Cao (Radix Glycyrrhizae), and Da Zao (Fructus Jujubae). Huang Qin Tang has been used for centuries in Asia to treat gastrointestinal disorders, nausea, and diarrhea.
Table 5 Bioactivities of TCM herbs. (Modeled after McCulloch et al. (2018))

\begin{tabular}{|c|c|}
\hline Bioactivity & TCM herbs \\
\hline Antiproliferative & $\begin{array}{l}\text { Bai Hua She She Cao } \\
\text { (Herba Oldenlandia), } \\
\text { Huang Qin (Scutellaria } \\
\text { baicalensis), Jiang Huang } \\
\text { (Rhizoma Curcumae } \\
\text { Longae, turmeric), Ling } \\
\text { Zhi (reishi mushroom or } \\
\text { Ganoderma) }\end{array}$ \\
\hline Apoptotic & $\begin{array}{l}\text { Jiang Huang (Rhizoma } \\
\text { Curcumae Longae, } \\
\text { turmeric), Green tea } \\
\text { (Camellia sinensis) }\end{array}$ \\
\hline $\begin{array}{l}\text { Inhibitive (NF-kappa B, } \\
\text { COX-2, telomerase) } \\
\text { Pulmonary fibrosis } \\
\text { (radiation-induced) }\end{array}$ & $\begin{array}{l}\text { Jiang Huang (Rhizoma } \\
\text { Curcumae Longae, } \\
\text { tumeric) } \\
\text { Green tea (Camellia } \\
\text { sinensis) } \\
\text { Dang Gui (Angelicae } \\
\text { sinensis) }\end{array}$ \\
\hline $\begin{array}{l}\text { Synergistic with } \\
\text { chemotherapy agents } \\
\text { (anthracycline, cisplatin, } \\
\text { and irinotecan) }\end{array}$ & $\begin{array}{l}\text { Green tea (Camellia } \\
\text { sinensis) }\end{array}$ \\
\hline $\begin{array}{l}\text { Reductive and preventive } \\
\text { (radiation-induced) } \\
\text { Enteritis } \\
\text { DNA damage } \\
\text { Pneumonitis }\end{array}$ & $\begin{array}{l}\text { Ren Shen (Radix Ginseng, } \\
\text { Panax ginseng) } \\
\text { Dang Gui (Radix } \\
\text { Angelicae sinensis, dong } \\
\text { quai) } \\
\text { Bai Shao (Radix Paeoniae } \\
\text { Alba) }\end{array}$ \\
\hline $\begin{array}{l}\text { Bone marrow and } \\
\text { intestinal toxicity }\end{array}$ & $\begin{array}{l}\text { Shu Di Huang (Radix } \\
\text { Rehmanniae Preparata) } \\
\text { and Chuanxiong (Rhizoma } \\
\text { Ligustici Chuanxiong) } \\
\text { Dong Chong Xia Cao } \\
\text { (Ophiocordyceps sinensis) }\end{array}$ \\
\hline
\end{tabular}

PHY906 has now been formulated as a powder containing a spray-dried aqueous extract derived from the four principal herbs. The research was undertaken by Professor Ying Chi Cheng, who co-founded with Yale University's Office of Cooperative Research, PhytoCeutica, a biopharmaceutical company dedicated to developing cancer drugs from TCM herbs. According to the National Cancer Institute's drug trial information site: “. . .PHY906 possesses a wide range of pharmacological activities such as the enhancement of oral uptake of pharmacologically active agents, inhibition of CYP3A4, modulation of certain 
cytokines, macrophages and lymphocytes, and inhibition of expression of MMP, NF-kB, betaglucuronidase, the NK-1 receptor, and the deltaopioid receptor" (Dillon 2007).

TCM has now been shown to be a clinical competitor of pharmaceutical drugs in efficacy with recent studies proving the bio-chemotherapeutic effects of synergistic herbal compounds in oncological applications. These same herbs are also proving superior ratings over standard of care in decreased adverse effects and toxicity levels.

The result of a German study on pancreatic cancer elimination with dietary and herbal compounds was published in 2014 in the International Journal of Oncology (Li et al. 2014).

The unique aspects of this study are the bioactivities of a collection of multiple agents found as superior to a singular agent in increasing apoptosis and remission and decreasing tumorigenicity and metastasis and the targeting of cancer stem cells as primary in elimination of advanced pancreatic ductal adenocarcinoma. PDA is a highly aggressive malignancy with poor prognosis given the current therapeutic options, which do not target cancer stem cells (CSCs), considered to be the central cause for the aggressiveness.

Polyphenols from dietary agents, sulforaphane and quercetin, along with the green tea catechin EGCG, resveratrol, genistein, and curcumin, hold promise as anti-CSC agents in PDA. In vitro data and mouse experiments suggest that sulforaphane eliminates pancreatic CSCs by inhibition of bioactive agents for cancer stem cells targeting NF- $\kappa \mathrm{B}$ activity and self-renewal potential and sensitizes the cells to apoptosis induction. The combination of dietary agents was found to be superior in reducing the self-renewal potential, viability, and migratory potential, along with increased induction of apoptosis.

In addition, the diterpenoid triepoxide triptolide was noted to be a promising combination partner. Triptolide has a long history in Traditional Chinese Medicine for the treatment of rheumatoid arthritis and cancer. It is an active compound of Lei Gong Teng (Tripterygium wilfordii), demonstrated in a prior study to effectively inhibit $\mathrm{NF}-\kappa \mathrm{B}$ activity, epithelial-mesenchymal transition, and stemlike features in PDA cells (Li et al.
2014). Previous studies have shown chemopreventive nutritional polyphenols, and isothiocyanates may neutralize genetic defects by epigenetic regulation, the potential reason why these substances attenuate the processes of tumorigenesis, progression and metastasis, and sensitization for drug treatment. Future studies will need to address an "epigenetic diet" created for the presence of selected bioactive agents for prevention and treatment of cancer (Appari et al. 2014).

The previous illustrarion (Fig. 6) details many of the study's findings regarding the links with genetics, epigenetics, and tumorigenicity and how cancer stem cells are biologically affected by the compounds found in TCM herbal medicines and dietary considerations.

The emerging sciences of nutrigenetics and nutrigenomics are embracing these concepts and developing strategies to educate healthcare professionals and patients as to the imperative of nutritional therapy in healthcare. The evidence contained in the study relating to the elimination of cancer stem cells by phytochemical influences should motivate advanced investigation for future cancer research and treatment protocols.

\section{Network Pharmacology and Systems Biology: The Next Generation of Herbal Medicine}

Aligned with the meridian concepts of TCM, network pharmacology includes regulation of the signaling pathway with multiple channels, upgraded drug efficacy, and higher success rates for clinical trials, as well as reduced adverse reactions and decreased budgetary considerations of pharmaceutical research and development. Network pharmacology also explores the parameters of how and where a singular target can inhibit or activate disease phenotypes.

This vital information can assist in developing therapies that are less vulnerable to drug resistance. These mechanisms can be accomplished by a synergistic effect, much like the TCM principles and practices that embrace a multivariate, collaborative approach. Network pharmacology also offers molecular interaction validation 


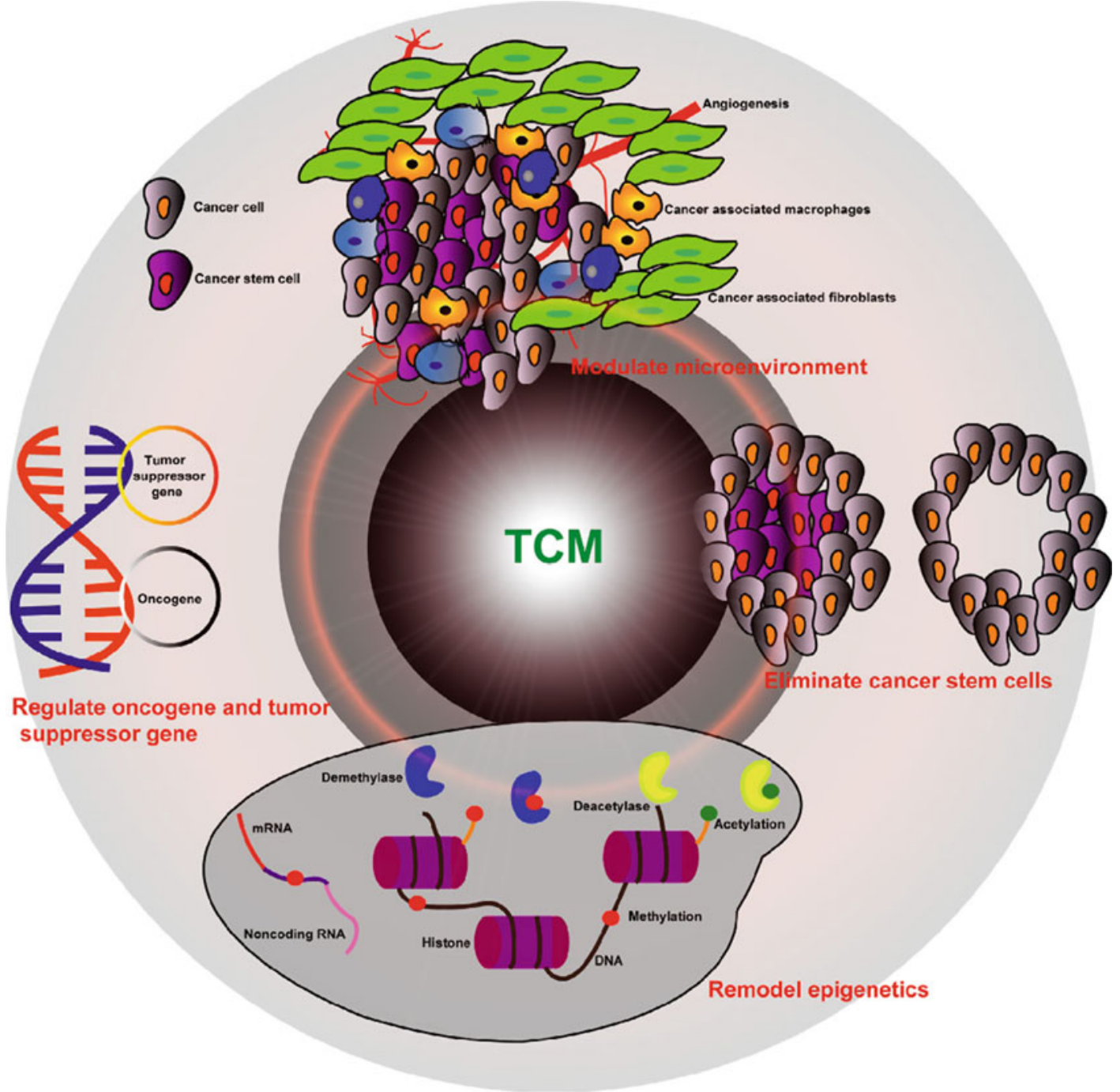

Fig. 6 Schematic diagram demonstrating the application of TCM in cancer therapy based on genetics, epigenetics, tumor microenvironment, and cancer stem cells. (Use Permission by Creative Commons Licensure 4.0: (C) 2019,
Yuening Xiang, Zimu Guo, Pengfei Zhu, Jia Chen, Yongye Huang Cancer Medicine published by John Wiley \& Sons Ltd) technology as a viable tool which reveals drug activity mechanisms and verifies the predictability of a drug network.

Owing to its origins to bioscience research, systems biology is a recent trend, providing a more holistic view on the complexity of biological systems interactions. This is a more encompassing perspective than isolating and altering a singular molecular component. Network pharmacology, as a systems biology-based methodology, replaces the standard accepted drug design of a solo pharmaceutical by investigating multiple activities that drugs may exert collectively on biological networks. There is a search for combining therapeutic drug protocols that are emerging in network pharmacology.

In allegiance with systems biology, network pharmacology is challenging the single-target drug research and development model in favor of a more comprehensive and collaborative approach. 
Over three decades of pharmaceutical development has produced hundreds of popularized and largely successful drugs. However, "the single-symptom, single-disease, single-drug concept" has not been as influential on certain degenerative diseases, including hypertension, depression, diabetes, inflammation, and cancer. While they may have marginal success in management, all too often, singular drug therapies are inadequate, and multiple drugs or increased dosages are recommended, causing an increase in adverse reactions and heightened toxicity, without achieving the needed metabolic therapeutic result (Kola and Landis 2004). Subsequent to these pharmaceutical pitfalls, the shift is toward a "network-target, multiple-component-therapeutics" mode (Li et al. 2013, 2014).

TCM exemplifies the natural order when approaching treatment protocols. Restoring balance is paramount, and reflecting the body's natural ability to heal is considered most valuable when choosing combinative herbal prescriptions. Network pharmacology is adopting some of these ancient concepts by utilizing TCM's wider lens on multiple action and reaction formulations and integrating the information of disease phenotypes, genetic influences, and biological molecules in a more integrated research model ( $\mathrm{Li}$ et al. 2013).

\section{TCM and Clinical Pharmacology In Silico}

One of the frontline advantages of network pharmacology and systems biology is the intimate partnership with bioscience informatics and multiple exhaustive global databases being constructed. A number of databases have emerged over the past decade, which bring TCM herbal medicine and network pharmacology to the same dialogue through informatics, algorithms, data mining, and extensively designed digital platforms. As an example, TCM-Mesh highlights TCM network pharmacology through cataloguing diseases, herbs, compounds, genes, proteins, and drugs into a comprehensive and cross-referenced biological digital data vault (Zhang et al. 2019). Other relevant network pharmacology and TCM databases include:

\section{Asian Anticancer Materia Database, Institute} of East-West Medicine, USA. This summarizes 700 kinds of anticancer drug information from Asia, of which $80 \%$ are derived from medicinal plants. This database also includes the commonly used Chinese medicine name, Latin name, medicinal properties, the major compounds, and other information (Yi F et al. 2018).

2. Chem-TCM, Institute of Pharmaceutical Science at King's College London, UK. This contains more than $350 \mathrm{TCMs}$ and over 9500 compounds. It records the compounds, related plants, chemical properties, common target activities, and other information.

3. Chinese National Compound Library, National Health and Family Planning Commission of the People's Republic of China. This is a library of small-molecule compounds consisting of core libraries and satellite libraries. It contains physical and chemical information of nearly two million small molecules.

4. CHMIS-C (Comprehensive Herbal Medicine Information System for Cancer), University of Michigan Medical School, USA. This provides 527 anticancer herb prescriptions, 937 components, and 9366 smallmolecule structures for the clinical treatment of different types of cancer, combined with a reference database and a molecular target aided database.

5. CNPD (Chinese Natural Products Database), Shanghai Institute of Materia Medica, Chinese Academy of Sciences, China. The CNPD database currently collects more than 57,000 natural products from 37 categories, of which $70 \%$ of the molecules are drug-like molecules. The relevant data include the CAS number, name, molecular formula, molecular weight, melting point, and other physical and chemical properties of natural products.

6. MAS3.0, CapitalBio, China. MAS (molecule annotation system) is a whole data mining and function annotation solution to extract and analyze biological molecule relationships from public knowledge base of biological molecules and signification. MAS analysis platform is a web client program for interactive navigation. MAS uses relational databases of biological 
networks created from millions of individually modeled relationships between genes, proteins, diseases, and tissues.

7. PharmMapper Server, Shanghai Institute of Materia Medica, China. PharmMapper Server is a freely accessed web server designed to identify potential target candidates for the given probe small molecules, drugs, natural products, or other newly discovered compounds with binding targets unidentified using pharmacophore mapping approach (Liu XF et al. 2010).

\section{Nobel Prize Honors TCM}

Qinghao (Herba Artemisiae Annuae), a TCM herb, provided the basis for an anti-malaria formula, since the protozoan had become resistant to quinine, an older medication used for treating the pathogen. This "new" discovery had been revealed in Ge Hong's A Handbook of Prescriptions for Emergencies (肘后备急方), an ancient medical and scientific document. In 1972, after screening over 2000 different Chinese herbs, phytochemist and researcher Youyou Tu and a cohort of scientists uncovered the exact formulation of artemisinin (qinghaosu 青蒿素) and dihydroartemisinin from Qinghao.

A graduate of the Department of Pharmaceutics at Beijing Medical College, Tu was chosen to join the China Academy of Chinese Medical Sciences and currently holds the highest ranking post of Chief Scientist in the Academy. According to Professor $\mathrm{Tu}$, malaria was one of the epidemic diseases with the most comprehensive records in TCM literature. She found a landmark quote for alleviating malaria fevers that motivated the research: "A handful of Qinghao immersed in two liters of water, wring out the juice and drink it all” (青蒿 一握，以水二升渍，绞取汁，尽服之). $\mathrm{Tu}$ experimented with lower heat during extraction using water, ethanol, and ether ester to arrive at dihydroartemisinin, which proved to be a clinically effective compound in curing malaria.

Due to the relentless efforts of Tu and her research team over several decades, more than 200 million malaria patients have received artemisinin or artemisinin combination therapies.
In 2015, Youyou Tu was awarded the Nobel Prize in Medicine with William C. Campbell and Satoshi Ōmura; this was the first time a Chinese scientist had won the coveted award.

\section{Perspective}

TCM originated at a time when there were no sophisticated instruments as there are today in medicine and science. Regardless, TCM evolved as a highly advanced system able to identify energetic patterns within the human body aligned with the energetic properties of nature.

A striking relationship between TCM and clinical pharmacology has been established through numerous international databases linking a myriad of herbal, phytochemical, molecular, cellular, genetic, and chemical components into a warehouse of possibilities for advanced pharmaceutical and nutraceutical development. A clear distinction remains, however, in the cultural identity of patents and intellectual property ownership of western pharmaceutical enterprises and the eastern mentality of a shared natural resource. TCM has never claimed nature as its private domain and is eager to share the stage with its global counterparts for the betterment of healthcare for humanity.

A global spotlight has been placed on TCM with the 2019 adoption of the ICD-11 and the endorsement of TCM as a global healthcare option. TCM practitioners, like western medical practitioners are concerned about evidence-based knowledge and quality standardization of herbal medicines. TCM is the focus of increasing research at prestigious medical and scientific institutes, and the results are statistically remarkable.

From reviewing the PubMed Central (PMC) (National Library of Medicine and the National Institutes of Health), as of March 2020, 20,600+ studies were noted on TCM, 32,000+ on acupuncture, and 30,900+ on Chinese herbal medicine published in peer-reviewed journals throughout the world.

This chapter has presented TCM with evidence-based research in relation to clinical pharmacology, including addressing viable treatment options for epidemic levels of cancer and 
infectious disease such as SARS and COVID-19. Equally important is TCM's unique ability to offer new ways of combating the rapidly progressing culture of drug-resistant bacteria and viruses, which is a result of rampant abuse of antibiotics over recent decades. It also provides ways to strengthen the body and increase innate immunity with TCM diet and nutrition, acupuncture, acupressure, Chinese herbal medicine, lifestyle changes and Qigong/Taichi exercises, which is ultimately the best prophylactic medicine to prevent diseases and infections.

Both TCM and clinical pharmacology are now at a vital crossroad with international medical and scientific proposals on herbal production and standardization, as well as advances in network pharmacology, phytopharmacology, systems biology, data mining, and informatics, among others. By joining forces, TCM and clinical pharmacology can accomplish together more than either can do on their own, providing preventive and holistic approaches with the goal of giving the best health care possible to people around the world.

Acknowledgments The author wishes to extend her most sincere and heartfelt appreciation to Contributing Editor, Lorna Zilba, MA and the following persons who have graciously shared their time and expertise in reviewing this chapter and offering valuable commentary:

John K. Chen, PhD, Pharm D., OMD, LAc

Elizabeth Chen Christenson, MD, LAc

Edward B. Christenson, MD, Dipl.ABA

Cheryl Schwartz, DVM

Robert M. Grant, MA

\section{References and Further Reading}

Afshin A et al (2019) Health effects of dietary risks in 195 countries, 1990-2017: a systematic analysis for the Global Burden of Disease Study 2017. Lancet 393:1958-1972

Appari M et al (2014) Sulforaphane, quercetin and catechins complement each other in elimination of advanced pancreatic cancer by miR-let-7 induction and K-ras inhibition. Int J Oncol 45:1391-1400

Bensky D (2015) Chinese herbal medicine: materia medica, 3rd edn. Eastland Press, Seattle

Boh B et al (2007) Ganoderma lucidum and its pharmaceutically active compounds. Biotechnol Annu Rev 13:265-301

Chen CJ, et al. (2008) Toona sinensis Roem tender leaf extract inhibits SARS coronavirus replication, J Ethnopharmacol Oct 30;120(1):108-11
Chen CY (2011) TCM database@Taiwan: the world's largest traditional Chinese medicine database for drug screening in silico. PLoS One 6:e15939. https://oi. org/10.1371/journal.pone.0015939

Chen JK, Chen TT (2004) Chinese medical herbology \& pharmacology. Art of Medicine Press, City of Industry

Chen, JK (2020) How COVID-19 (2019-nCoV) is Currently Treated in China with TCM, https://www.elotus. org/article/how-covid-19-2019-ncov-currently-treatedchina-tcm

Cragg GM, Newman DJ, Snader KM (1997) Natural products in drug discovery and development. J Nat Prod 60:52-60

Dillon J (2007) From the potion to the pill. Yale Medicine. https://medicine.yale.edu/news/yale-medicine-maga zine/from-the-potion-to-the-pill/

Duke-Margolis, Center for Health Policy, FDA (2018) Identifying the root causes of drug shortages and finding enduring solutions. Duke University and FDA Presentation, Washington, DC. https://healthpolicy.duke. edu/sites/default/files/atoms/files/dukefda_drug_short ages_presentation_slides_0.pdf

Eban K (2019) Bottle of lies: the inside story of the generic drug boom. Harper Collins, New York

Ekor M (2014) The growing use of herbal medicines: issues relating to adverse reactions and challenges in monitoring safety. Front Pharmacol 4:177

Fabricant DS, Farnsworth NR (2001) The value of plants used in traditional medicine for drug discovery. Environ Health Perspect 109(Suppl 1):69-75

Fan X et al (2011) Network toxicology and its application to traditional Chinese medicine. Zhongguo Zhong Yao Za Zhi 36:2920-2922

Fan AY et al (2017) Acupuncture's role in solving the opioid epidemic: evidence, cost-effectiveness, and care availability for acupuncture as a primary, non-pharmacologic method for pain relief and management. J Integr Med 15(6):411-425. https://doi.org/ 10.1016/S2095-4964(17)60378-9

Hamburger M, Hostettmann K (1989) Analytical aspects of drugs of natural origin. J Pharm Biomed Anal 7:1337-1349

He LS et al (2016) Administration of traditional chinese blood circulation activating drugs for microvascular complications in patients with type 2 diabetes mellitus. J Doabetes Res 2016:1081657

Ideker $\mathrm{T}$ et al (2001) A new approach to decoding life: systems biology. Annu Rev Genomics Hum Genet 2:343-372

Jia DD (1979) Concise history of Chinese medicine (Zhong Guo Yi Xue Shi Lue). Shanxi People's Press, Taoyuan

Jiang WY (2005) Therapeutic wisdom in traditional Chinese medicine: a perspective from modern science. Trends Pharmacol Sci 26:558-563

Kola I, Landis J (2004) Can the pharmaceutical industry reduce attrition rates? Nat Rev Drug Discov 3:711-715

Lei X et al (2015) Liver damage associated with Polygonum multiflorum Thunb.: a systematic review of case reports and case series. Evid Based Complement Alternat Med 2015:459749. Epub 2015 Jan 12. Review 
Li S, Zhang B (2013) Traditional Chinese medicine network pharmacology: theory, methodology and application. Chin J Nat Med 11:110-120

Li S et al (2011) Network target for screening synergistic drug combinations with application to traditional Chinese medicine. BMC Syst Biol 5 (Suppl 1):S10

Li QY et al (2013) Network pharmacology: a new approach for Chinese herbal medicine research. Evid Based Complement Alternat Med 2013:1-9

Li $\mathrm{S}$ et al (2014) Network pharmacology in traditional Chinese medicine. Evid Based Complement Alternat Med 2014:138460

Liang X et al (2014) A novel network pharmacology approach to analyse traditional herbal formulae: the Liu-Wei-Di-Huang pill as a case study. Mol BioSyst 10:1014-1022

Light D (2014) New prescription drugs: a major health risk with few offsetting advantages new prescription drugs: Harvard-Edmond J. Safra Center for Ethics. https:// ethics.harvard.edu/blog/new-prescription-drugs-majorhealth-risk-few-offsetting-advantages

Liu XF, et al (2010) PharmMapper server: a web server for potential drug target identification using pharmacophore mapping approach, Nucleic Acids Research, Nucleic Acids Research, 38(suppl_2):W609-W614.

Liu L et al (2014) Triptolide reverses hypoxia-induced epithelial-mesenchymal transition and stem-like features in pancreatic cancer by $\mathrm{NF}-\kappa \mathrm{B}$ downregulation. Int J Cancer 134:2489-2503

Ma Y et al (2019) Prevention and treatment of infectious diseases by traditional Chinese medicine: a commentary. https://onlinelibrary.wiley.com. 24 May 2019

Maciocia G (2015) The foundations of Chinese medicine, 3rd edn. Hardcover, Churchill Livingstone, London. ISBN 9780702052163

McCulloch M, Marshall AC, Nachat A (2018) Modern thoracic oncology, vol 1. World Scientific, New Jersey, pp 261-267

McDonald J, Janz S (2017) Acupuncture evidence project: a comparative literature review. Australian Acupuncture and Chinese Medicine Association, Brisbane, p 181. http://www.acupuncture.org.au/ OURSERVICES/Publications/AcupunctureEviden ceProject.aspx

Rates SMK (2001) Plants as source of drugs. Toxicon 39:603-613
Sperber G, Flaws B (2007) Integrated pharmacology: combining modern pharmacology with Chinese medicine. Blue Poppy Press, Boulder

Swinney DC, Anthony J (2011) How were new medicines discovered? Nat Rev Drug Discov 10(7):507-519

Veeresham C (2012) Natural products derived from plants as a source of drugs. J Adv Pharm Technol Res 3 (4):200-201

WHO (2002) Acupuncture: review and analysis of reports on controlled clinical trials. WHO, Geneva

WHO (2004) SARS: Clinical trials on treatment using a combination of Traditional Chinese Medicine and Western Medicine. http://apps.who.int/medicinedocs/ en/d/Js6170e/4.html

WHO (2014) WHO traditional medicine strategy 2014-2023. World Health Organization. https://www. who.int/medicines/publications/traditional/trm_strat egy14_23/en/

WHO (2018) Newsroom health topics. World Health Organization. https://www.who.int/news-room/fact-sheets/ detail/cancer

Williamson E et al (1996) Selection, preparation and pharmacological evaluation of plant material. Wiley, Chichester

Wright CW et al (2010) Ancient Chinese methods are remarkably effective for the preparation of artemisinin-rich extracts of Qing Hao with potent antimalarial activity. Molecules 15(2):804-812

Xiang Y et al (2019) Traditional Chinese medicine as a cancer treatment: modern perspectives of ancient but advanced science. Cancer Med 8:1958-1975. Published by Wiley

Yi F et al (2018) In silico approach in reveal traditional medicine plants pharmacological material basis. Chin Med 13:33

Zeng ZP Jiang JG (2010) Analysis of the adverse reactions induced by natural product-derived drugs. $\mathrm{Br} \mathrm{J}$ Pharmacol 159(7):1374-1391

Zhang R et al (2017) TCM-Mesh: the database and analytical system for network pharmacology analysis for TCM preparations. Sci Rep 7:2821

Zhang R et al (2019) Network pharmacology databases for traditional Chinese medicine: review and assessment. Front Pharmacol 10:123

Zhou X et al (2019) Current status and major challenges to the safety and efficacy presented by Chinese Herbal Medicine. Medicines (Basel) 6(1):1 